Livraisons

d'Histoire

de l'Architecture

\section{Livraisons de l'histoire de l'architecture}

28 | 2014

Jean-Louis Pascal

\title{
Les restaurations de Pascal à la Bibliothèque nationale : les ailes nord et est de la cour d'honneur
}

Pascal's restorations at the National Library: the Northern and Eastern Wings of the Honour Courtyard

Die Restaurierungen Pascals an der Bibliothèque Nationale: die Nordflügel und der Ehrenhof

\section{Anne Richard-Bazire}

\section{(2) OpenEdition}

Journals

\section{Édition électronique}

URL : http://journals.openedition.org/lha/394

DOI : 10.4000/lha.394

ISSN : 1960-5994

Éditeur

Association Livraisons d'histoire de l'architecture - LHA

Édition imprimée

Date de publication : 10 décembre 2014

Pagination : $99-128$

ISSN : 1627-4970

\section{Référence électronique}

Anne Richard-Bazire, "Les restaurations de Pascal à la Bibliothèque nationale : les ailes nord et est de la cour d'honneur », Livraisons de l'histoire de l'architecture [En ligne], 28 | 2014, mis en ligne le 10 décembre 2016, consulté le 19 avril 2019. URL : http://journals.openedition.org//ha/394 ; DOI : 10.4000//ha.394

Ce document a été généré automatiquement le 19 avril 2019.

Tous droits réservés à l'Association LHA 


\title{
Les restaurations de Pascal à la Bibliothèque nationale : les ailes nord et est de la cour d'honneur
}

\author{
Pascal's restorations at the National Library: the Northern and Eastern Wings of \\ the Honour Courtyard \\ Die Restaurierungen Pascals an der Bibliothèque Nationale: die Nordflügel \\ und der Ehrenhof
}

Anne Richard-Bazire

1 «Certains [architectes] veulent faire table rase et tout réinventer, de la structure de l'immeuble à son décor, d'autres pensent que l'on peut renouveler l'architecture grâce à un autre regard sur le passé $»^{1}$. Jean-Louis Pascal est l'un des premiers architectes à adopter une attitude nouvelle à l'égard de ce que l'on n'appelle pas encore le patrimoine. En même temps que sa nomination à la bibliothèque nationale, il obtient le poste d'architecte diocésain de Valence ${ }^{2}$ et restaure à ce titre la basilique Saint-Apollinaire. Le devis que Pascal rédige alors pour la réfection de l'intérieur de l'église montre le respect qu'il a de l'édifice original, de ses matériaux et des techniques de construction anciennes ${ }^{3}$.

2 Il aura le même souci à la Bibliothèque, respectant en tout point les volontés d'Henri Labrouste, tout au moins pour les travaux engagés par lui et « réservant seulement son indépendance pour les projets d'avenir qui ne résulteraient pas directement du plan d'ensemble accepté, il y a de longues années pour la restauration de la Bibliothèque nationale $»^{4}$.

\section{L'aile nord de la cour d'honneur (1878) : le refus d'une table rase du passé (ill. 1)}


III. 1 : Aile nord de la cour d'honneur de la Bibliothèque nationale

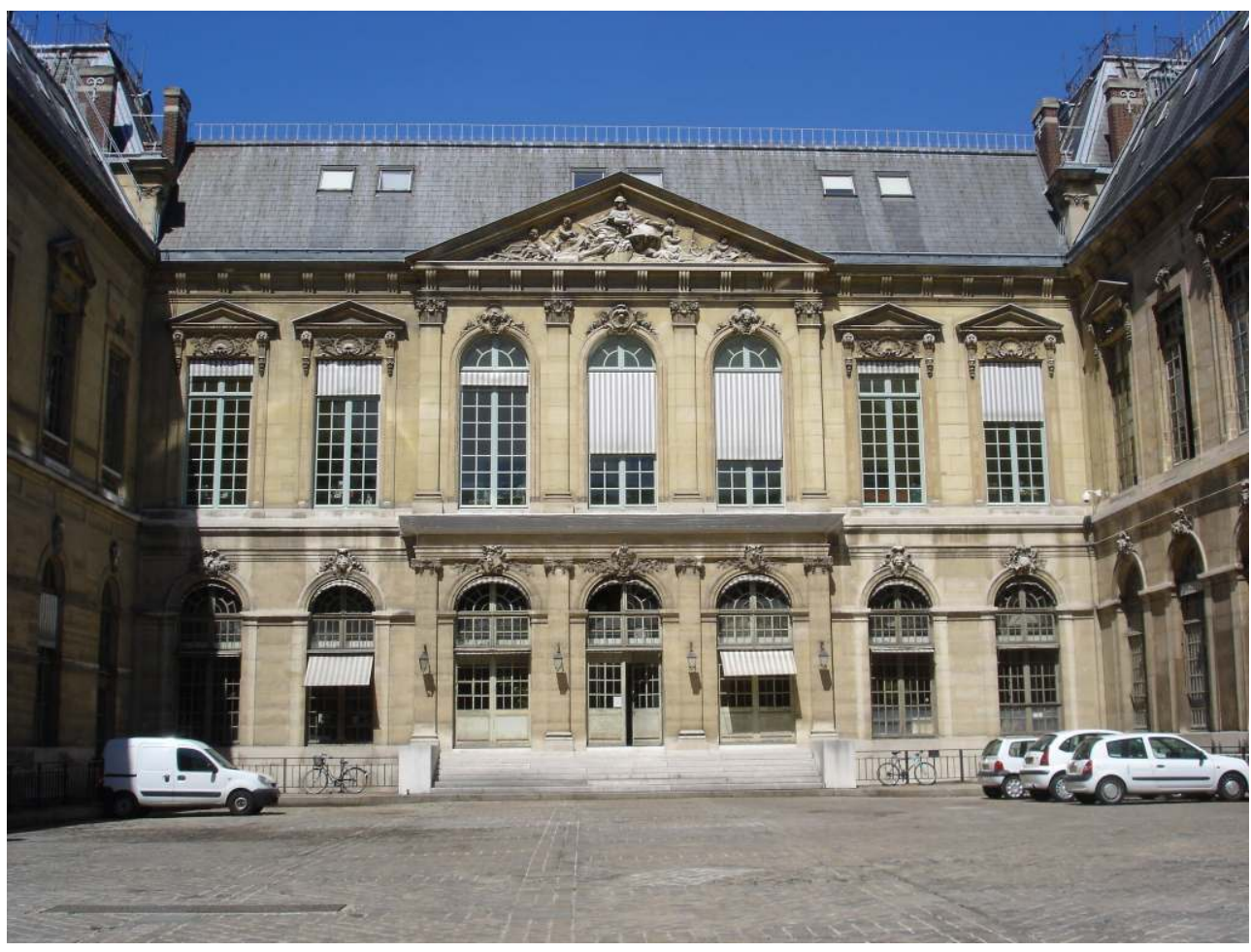

Architectes Jules-Robert de Cotte (1740-1749) et Jean-Louis Pascal (1875-1879)

Cl. auteur

3 Le mauvais état de cette ancienne construction posait le problème de sa démolition. La façade sur la cour d'honneur présentait une convexité de $0,17 \mathrm{~m}$. de flèche, provenant de la poussée des voûtes, qui étaient rompues en plusieurs endroits et avaient dû être étayées. Le mur de façade sur la rue Colbert, mur d'une épaisseur de 0,65 m., était formé de deux parements en moellons de petit appareil, de 0,16 m. d'épaisseur, reliés par des chaînes ; l'intervalle compris entre ces deux parements était rempli de gravats vidés à la hotte. Ce corps de bâtiment répondait fort mal, par ses dispositions intérieures, à l'usage auquel il était affecté : les livres qui y étaient emmagasinés se logeaient difficilement dans les pièces voûtées du rez-de-chaussée et dans un comble d'une énorme hauteur, encombré par une forêt de charpentes ${ }^{5}$.

4 Dans un rapport daté du 22 juillet 1874, Henri Labrouste en avait demandé la destruction. Il était en effet peu raisonnable de conserver une construction dans cet état et sa restauration aurait entraîné des dépenses exagérées. À la suite d'un rapport de Louis Duc, inspecteur général des bâtiments civils, attestant du mauvais état des bâtiments, le conseil des bâtiments civils conclut sa séance du 18 août 1874 en disant "que tous les travaux, qu'on pourrait proposer pour une consolidation quelconque, seraient plutôt nuisibles qu'utiles et qu'il convenait de laisser le bâtiment dans l'état où il se trouvait, attendu que rien ne faisait présumer qu'il ne pourrait attendre l'époque peu éloignée de sa reconstruction $»^{6}$.

5 La démolition du bâtiment fut alors commencée par Henri Labrouste et interrompue à la demande de l'administrateur Jules Antoine Taschereau ${ }^{7}$ et de son secrétaire Henri Delaborde ${ }^{8}$. Ce qui fera dire à Léon Labrouste : 
«Pourquoi faut-il après cette décision, après la démolition faite, que des influences d'amateurs aient fait revenir sur le jugement porté ? [...] On a doté Paris d'un second exemplaire de la faute commise au Louvre dans la construction de la colonnade de Perrault, en faisant des deux façades d'un même bâtiment des productions sans aucun rapport, bien qu'exprimant un seul et même besoin. Encore à la colonnade du Louvre avait-on l'excuse de garder debout une des façades existantes et de construire en style du XVII ${ }^{\mathrm{e}}$ siècle sous Louis XIV $»^{9}$.

Il faut dire que «la conscience patrimoniale progresse fortement pendant le Second Empire, et peu à peu [...] des voix s'élèvent pour déplorer des disparitions que nul n'aurait relevées vingt ans auparavant $»^{10}$. Vingt ans plus tard, cette conscience patrimoniale sera très forte, en témoignent les propos d'Henri Bouchot, conservateur à la Bibliothèque nationale :

«Si l'œuvre de de Cotte eût été condamnée en principe, personne mieux que Labrouste ne méritait le choix qu'avait fait la direction des bâtiments. Le nouvel architecte était de l'école des amoureux de l'antique, et les décorations imaginées plus d'un siècle auparavant lui paraissaient autant d'hérésies, de choses choquant le bon goût et la science architecturale. Il eut ce rêve de détruire à jamais la besogne de son illustre prédécesseur et de lui substituer l'ordonnance raide ou engoncée des pseudo-grecs [...] "Ce Vandale", pour employer l'expression de M. Paul Mantz, promena son œuvre de destruction en chaque chose, trouvant son excuse dans la substitution du fer au bois, sous le faux prétexte donné à entendre de restreindre ainsi les causes d'incendie $»^{11}$.

Jean-Louis Pascal estimait qu'il fallait conserver les vieux bâtiments. L'état lamentable des maçonneries de l'aile nord obligeait à les refaire entièrement pour conserver la façade sur cour. Dans une lettre au ministre des travaux publics, il écrit :

«M. Labrouste avait décidé pour cette partie la démolition complète des restes encore existants de la façade sur la cour, spécimen de l'architecture du siècle dernier et témoin intéressant au point de vue historique et archéologique. La partie gauche de ce bâtiment est en effet déjà refaite en partie (ill. 2), conformément aux autres côtés reconstruits de la cour, et il ne serait possible de conserver auprès de l'architecture nouvelle, l'avant-corps couronné d'un fronton, qui faisait l'axe de la façade ancienne, qu'en substituant aussi à sa droite une reproduction des motifs de Labrouste. Or dans ce cas on verrait la grosse corniche de l'ancienne partie, la mouluration, les attributs, les motifs du siècle dernier pénétrer au milieu de l'architecture très personnelle qui pourtourne la cour nouvelle. Une seconde solution (ill. 2) se présente : démolir la travée faite par Labrouste et l'ancienne partie qui lui fait pendant (pour la reculer à l'alignement de la première) et rétablir en arrière, deux nouveaux morceaux de façade, reproduisant l'ancienne architecture dont un fragment a été démoli $»^{12}$. 


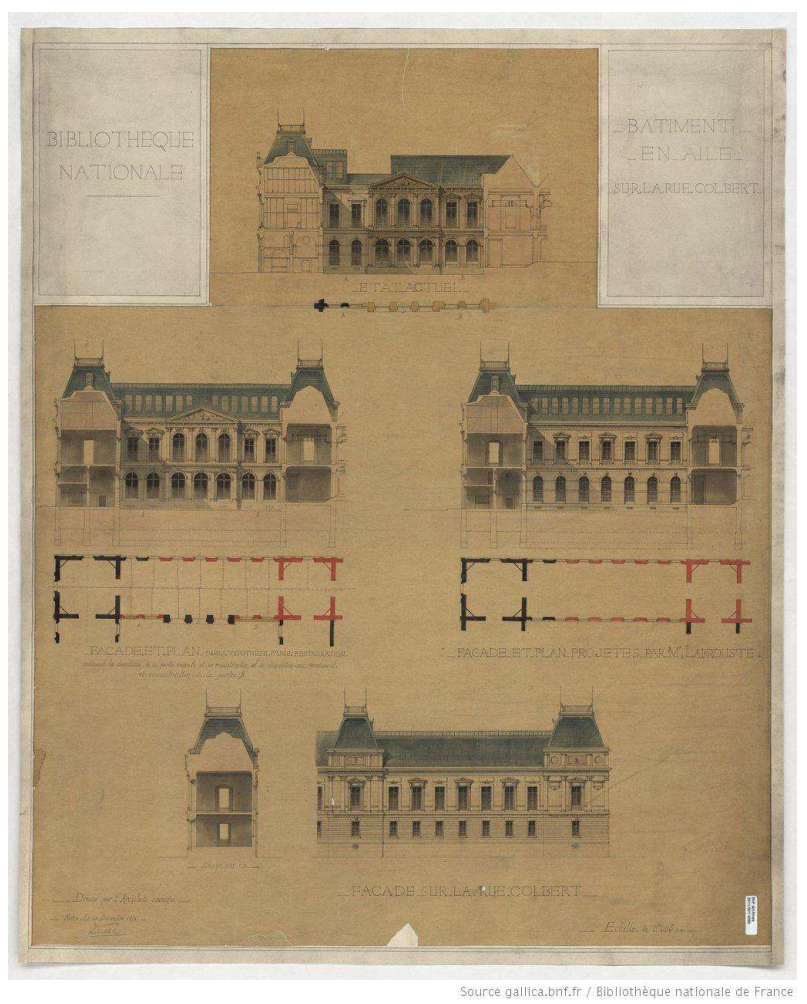

En haut : état actuel (1876) de la façade sur la cour d'honneur avec la partie gauche déjà reconstruite par Henri Labrouste. Au milieu à gauche : proposition de démolition de la partie neuve A (à gauche du fronton) et sa reconstruction, et de démolition avec reculement et reconstruction de la partie $\mathrm{B}$ (à droite du fronton). Au milieu, à droite : la façade telle qu'elle était projetée par Henri Labrouste. En bas, façade sur la rue Colbert. Jean-Louis Pascal. 23 décembre 1876. Dessin aquarellé sur calque collé sur papier. $680 \times 840 \mathrm{~mm}$.

Arch. adm. B.n.F., 2011/001/4093.

Cette seconde solution était coûteuse. L'inspecteur général Louis Duc fit observer à JeanLouis Pascal qu'un parti mixte, rappelant suffisamment l'ancienne disposition architecturale, mais composé des éléments nouveaux introduits par Henri Labrouste et respectant ainsi l'unité nécessaire à l'ensemble de la cour, donnerait peut-être satisfaction aux personnes regrettant surtout de l'ancienne façade sa variété, ses saillies, les mouvements de ses lignes ${ }^{13}$. Pascal proposa, en gardant la donnée générale et les dimensions de l'ancien avant-corps, deux variantes (ill. 3) dont les motifs étaient empruntés aux façades nouvelles. 


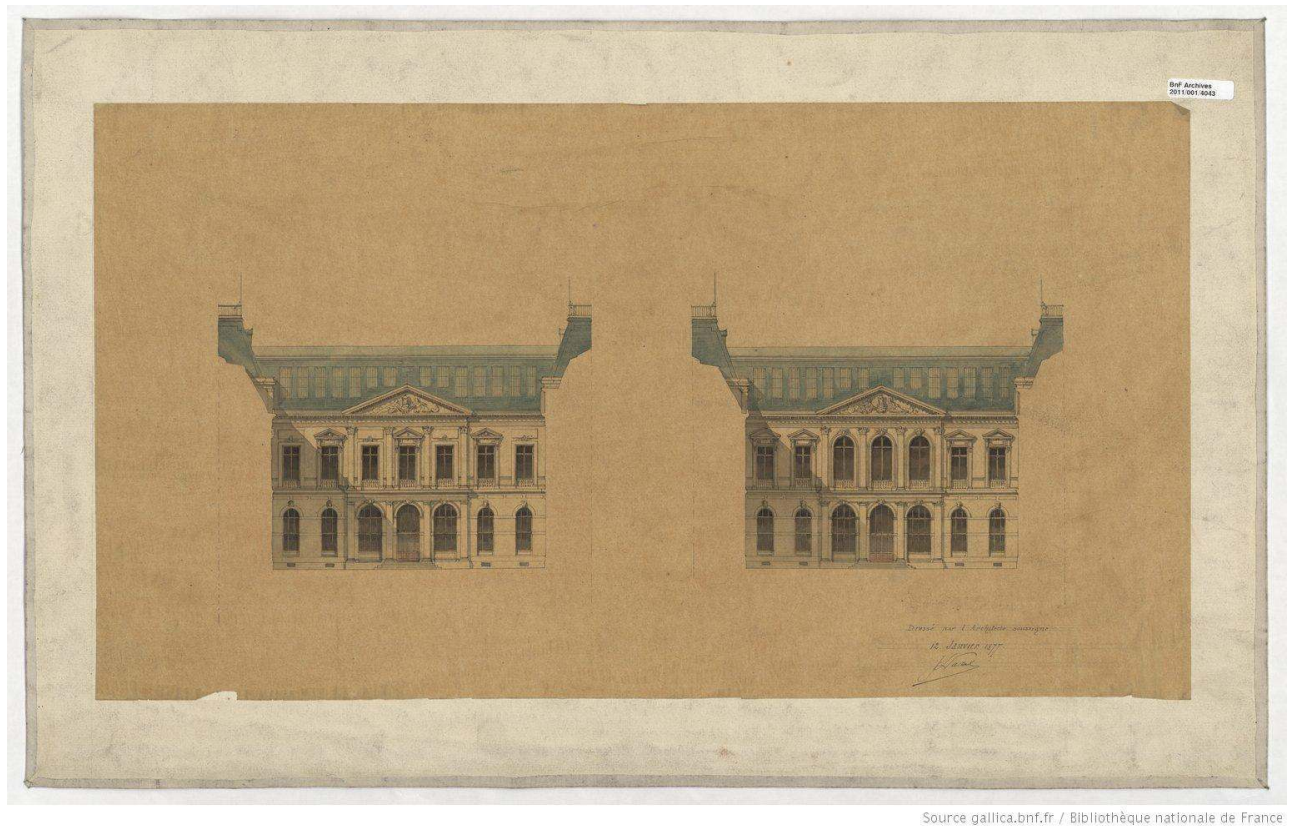

Restauration et aménagement, projet. Jean-Louis Pascal. 12 janvier 1877. Dessin aquarellé sur calque collé sur papier. 402 × $650 \mathrm{~mm}$

Arch. adm. B.n.F., 2011/001/4043

Dans une première version, il reprit l'ordonnance des pilastres de Labrouste, la corniche fine à modillons, les fenêtres modernes. Dans une seconde variante, il reconstitua un motif plus semblable à l'ancienne façade avec trois fenêtres cintrées au premier étage.

Jean-Louis Pascal était partagé: le moyen terme avait pour avantage matériel de conserver complètement l'amorce déjà bâtie par Henri Labrouste et d'épargner les combinaisons compliquées de reconstruction. Il permettait de conserver dans la cour trois côtés de même caractère et d'en laisser subsister un seul comme spécimen de l'ancien édifice ${ }^{14}$. "Une des raisons surtout pour maintenir la solution de Labrouste de faire une architecture nouvelle est l'effet bizarre qu'aurait présenté un rectangle bâti dont deux côtés de chaque angle opposé présente une architecture différente ${ }^{15}$. Le rapport de Pascal s'achevait ainsi : "D'autres variantes pourraient être proposées mais elles dérivent de ce point de départ de conserver de l'ancienne façade l'avant-corps et le fronton en l'habillant avec des formes qui dominent maintenant à la bibliothèque. Moyen mixte qui a pour moi le mérite de respecter l'œuvre commencée de mon prédécesseur sans trop choquer les sentiments respectables qui plaident en faveur de la conservation des vieilles formes $»^{16}$.

11 Un rapport sur les propositions de Pascal est alors fait au conseil des bâtiments civils par Duc, inspecteur général :

«L'architecte a introduit à la place de l'ordre du rez-de-chaussée des antes accompagnées de décorations accessoires qui s'éloignent absolument des anciens bâtiments [ce qui fait] craindre un désaccord de style de l'ordre du rez-de-chaussée avec celui du premier étage et surtout avec celui qui commande l'ancienne et principale façade de la cour [...] On voit en effet des antes sur la façade opposée construite par M. Labrouste [...] Je crois que M. Pascal a respecté trop scrupuleusement ce style qui avait peut-être sa raison d'être dans une façade qui pourrait s'affranchir de l'ordonnance régulière de la cour [...] Je serais donc d'avis 
de répéter, pour le rez-de-chaussée de la nouvelle façade à construire, l'ancien ordre ionique qui existait, en évitant de reproduire les cartouches flamboyants qui décoraient les clés des arcades et en y substituant des têtes : on obtiendrait ainsi un ensemble bien proportionné avec l'ordre corinthien du premier étage ${ }^{17}$.

Après une visite sur place, le conseil conclut à «l'impossibilité de concilier la réunion de deux styles » et son avis est encore plus radical encore que celui de Duc : «Il y a lieu de reproduire, telle qu'elle existait autrefois, la façade intérieure du bâtiment sur la rue Colbert ». Ce qui fera dire à Léon Labrouste, qui avait travaillé douze ans à la Bibliothèque avec son père : «On a reconstruit en 1878, en plein XIX siècle, une façade démolie qui n'est plus qu'un décor, et ce décor n'ayant de méritant que la copie d'un fronton qu'il était possible de placer sur l'ancien bâtiment restauré du fond de la cour d'honneur $»^{18}$.

Jean-Louis Pascal démolit donc les travées amorcées par Labrouste sur la façade nord de la cour d'honneur et y rétablit l'ordonnance de Jules-Robert de Cotte. Il proposa ${ }^{19}$, pour reproduire le fronton qui représente Minerve promenant son compas sur le globe terrestre ${ }^{20}$, le sculpteur Charles Degeorge qui avait réalisé le buste d'Henri Regnault dans la cour du mûrier à l'École des beaux-arts :

«Le fronton était la partie la plus remarquable de cette construction; il a été démoli en 1878 et on a refait une copie. Admirablement composé, d'une exécution simple et large, il représentait Minerve, la tête couverte d'un casque surmonté d'un hibou ; à ses pieds était l'égide aux armes de la France, elle était entourée de génies accompagnés des attributs des arts, des lettres, des sciences et du commerce. $»^{21}$

C'est Auguste Perrin à qui l'on doit toutes les sculptures décoratives de la Bibliothèque exécutées sous la direction et d'après les dessins d'Henri Labrouste. Il meurt en 1878. Son gendre, le sculpteur Léon Auguste Perrey ${ }^{22}$ (1841-1900) avait contribué pour une large part à l'exécution de ces travaux. Pascal propose de lui confier toutes les parties de sculpture qui reproduisent des modèles appartenant au type d'architecture d'Henri Labrouste, c'est-à-dire toute la façade du pavillon et du corps de bâtiment donnant sur la rue Colbert (ill. 2) ; mais pour une parfaite reconstitution archéologique de la façade sur la cour d'honneur, Pascal choisit des sculpteurs avec lesquels il a déjà travaillé :

«Pour la seconde partie, c'est-à-dire pour la façade sur la cour, qui ne doit être que la reproduction de la façade de Robert de Cotte, récemment démolie, il m'a paru que des artistes d'une compétence spéciale assureraient plus complètement une exécution d'un caractère quasi archéologique et je proposerais l'admission de M.M. Watrinelle et Germain demeurant Boulevard d'Enfer, 59, et 13 rue Boissonade, dont j'ai pu apprécier le talent, soit dans des travaux particuliers, soit pour des modèles exécutés pour le duc d'Aumale à Chantilly ${ }^{23}$ sous la direction de M. Daumet, soit enfin pour une reproduction de motifs décoratifs de nature analogue, quoique d'un style tout différent, que j'ai eu à faire exécuter pour l'exposition portugaise au Champ de $\operatorname{Mars}^{24}$ [...] Reste une partie plus spécialement artistique: c'est la reproduction du fronton de cette façade dont j'avais heureusement fait prendre un moulage, reproduction qui ne peut être confiée qu'à un statuaire. Je propose de donner l'exécution à $\mathrm{M}$. Degeorge pour le prix de trois mille cinq cents francs ${ }^{25}$.

Antoine Watrinelle (1818-1913) et Gustave Germain (1843-1909) ${ }^{26}$, vont alors procéder à des moulages ${ }^{27}$ de la façade donnant sur la cour d'honneur : «Les travaux ci-dessus indiqués seront exécutés par nous avec le plus grand soin et en respectant le côté archéologique des moulages exécutés ${ }^{28}$. Ils s'engagent à réaliser toute la sculpture de la façade sur cour pour 17000 francs ; Perrey, sculpteur ornemaniste, s'engage à exécuter toutes les parties de sculpture de la façade du pavillon et du corps de bâtiment donnant sur la rue Colbert pour 4500 francs ; Degeorge, sculpteur statuaire, s'engage à exécuter la 
reproduction du grand motif destiné à décorer le fronton du bâtiment de la cour d'honneur moyennant 3200 francs $^{29}$. Celui-ci sera achevé le 25 janvier 1880.

Profitant de cette reconstruction, Pascal avance l'ensemble de l'aile vers le sud de manière à élargir la rue Colbert et réussit un raccord parfait avec la façade est de la même cour. Pour la façade extérieure sur la rue Colbert, Jean-Louis Pascal reprend les motifs de son prédécesseur ; Léon Labrouste s'en félicitait, au nom de la mémoire de son père : « Sur la rue Colbert, le grand mur triste et nu qui bordait le bâtiment disparu et auquel étaient adossés de modestes étalages de librairie en plein vent, va être remplacé par une façade monumentale, de même ordonnance que la façade en retour sur la place Louvois $»^{30}$.

17 À l'intérieur, la nouvelle construction est destinée à recevoir provisoirement la salle publique de lecture située alors dans les bâtiments du fond de la cour d'honneur qui avaient besoin d'être restaurés ${ }^{31}:$ «La grosse construction du nouveau bâtiment sur la rue Colbert est maintenant terminée [dira Pascal en octobre 1879] [...] J'ai décidé que la salle de lecture publique qui occupe encore actuellement le $1^{\text {er }}$ étage du vieux bâtiment à reprendre serait transférée provisoirement au même niveau de la rue Colbert, l'entrée actuelle continuant à servir, en créant un nouvel escalier temporaire dans le petit pavillon voisin du concierge $»^{32}$

\section{Création d'une salle publique de lecture au $1^{\text {er }}$ étage de l'aile nord (1881)}

18 La première salle publique de lecture que Louis Visconti avait mise en place en 1833, au premier étage de l'aile est de la cour d'honneur, fut victime de son succès, car, chauffée et confortable, elle souffrit assez vite de son abondante - et pas toujours recommandable fréquentation ${ }^{33}$. Prosper Mérimée, sénateur et écrivain reconnu, dans le rapport ${ }^{34}$ qu'Achille Fould $^{35}$ (1800-1867) lui commanda en 1858 pour améliorer le fonctionnement de la Bibliothèque, protestait contre la trop large ouverture des Imprimés qui recevaient deux cent cinquante lecteurs par jour pour cent places seulement et demandait, à côté d'une salle réservée aux "travailleurs sérieux », la future "salle Labrouste », une salle d'accès libre pour deux cents personnes, pourvue de livres usuels non précieux. L'ouverture de la salle publique de lecture, ordonnée par l'article 6 du décret du 14 juillet 1858, fut fixée au 2 juin 1868, soit dix jours avant la grande salle de travail. Son utilité est telle, qu'en 1877, elle reçoit près de cinquante-neuf mille lecteurs empruntant quatrevingt-dix mille livres ${ }^{36}$. C'était une salle "populaire» mêlant ouvriers et étudiants, accessible à toute personne d'au moins seize ans, et gratuite. La salle Labrouste, par comparaison, était surnommée la "salle aristocratique»; il fallait une carte pour y rentrer. La salle publique était ouverte tous les jours de la semaine, à neuf heures; elle fermait à dix-huit heures en été et à seize heures en hiver ${ }^{37}$. Elle proposait un fonds de vingt-cinq mille volumes, livres classiques de littérature, d'histoire, d'ouvrages de technologie appliquée aux arts et métiers, de médecine et de droit.

En 1876, on arrange le rez-de-chaussée du pavillon Colbert en dépendance de la salle publique de lecture. Abritée tout d'abord dans le bâtiment du fond de la cour d'honneur, on y accédait par le $n^{\circ} 3$ de la rue Colbert (ill. 4), cette salle de lecture publique fut transférée et inaugurée en janvier 1881 au premier étage de l'aile nord (ill. 5) restituée par Jean-Louis Pascal qui écrit au ministre des travaux publics le 20 février $1881^{38}:$ «Le bâtiment sur la rue Colbert est livré à l'administration de l'établissement, ou tout au moins la salle de lecture du $1^{\text {er }}$ étage et les combles qui lui servent de dépôt ainsi que les 
caves qui contiennent le calorifère. Le rez-de-chaussée fort avancé pourra être livré à son tour dans quelques mois : on achève le montage des casiers. »

\section{4 : Plan général de la Bibliothèque nationale}

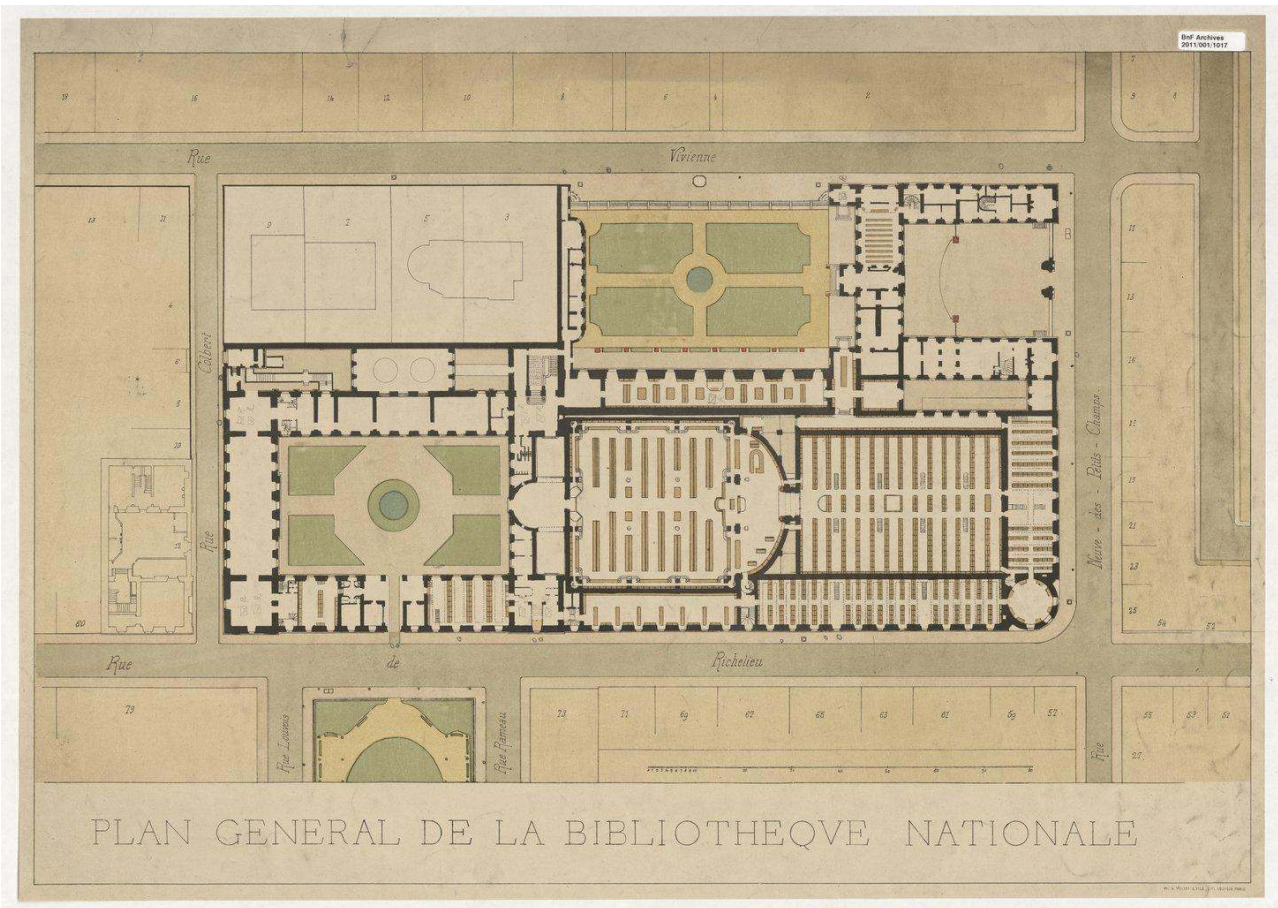

Source gallica.bnf.fr / Bibliothècue nationale de France

Rez-de-chaussée. 1881. Jean-Louis Pascal. Dessin aquarellé sur papier. 460 × 650 mm Arch. adm. B.n.F., 2011/001/1017 
III. 5 : La nouvelle salle de lecture publique inaugurée en 1881 au $1^{\text {er }}$ étage de l'aile Colbert de la Bibliothèque nationale

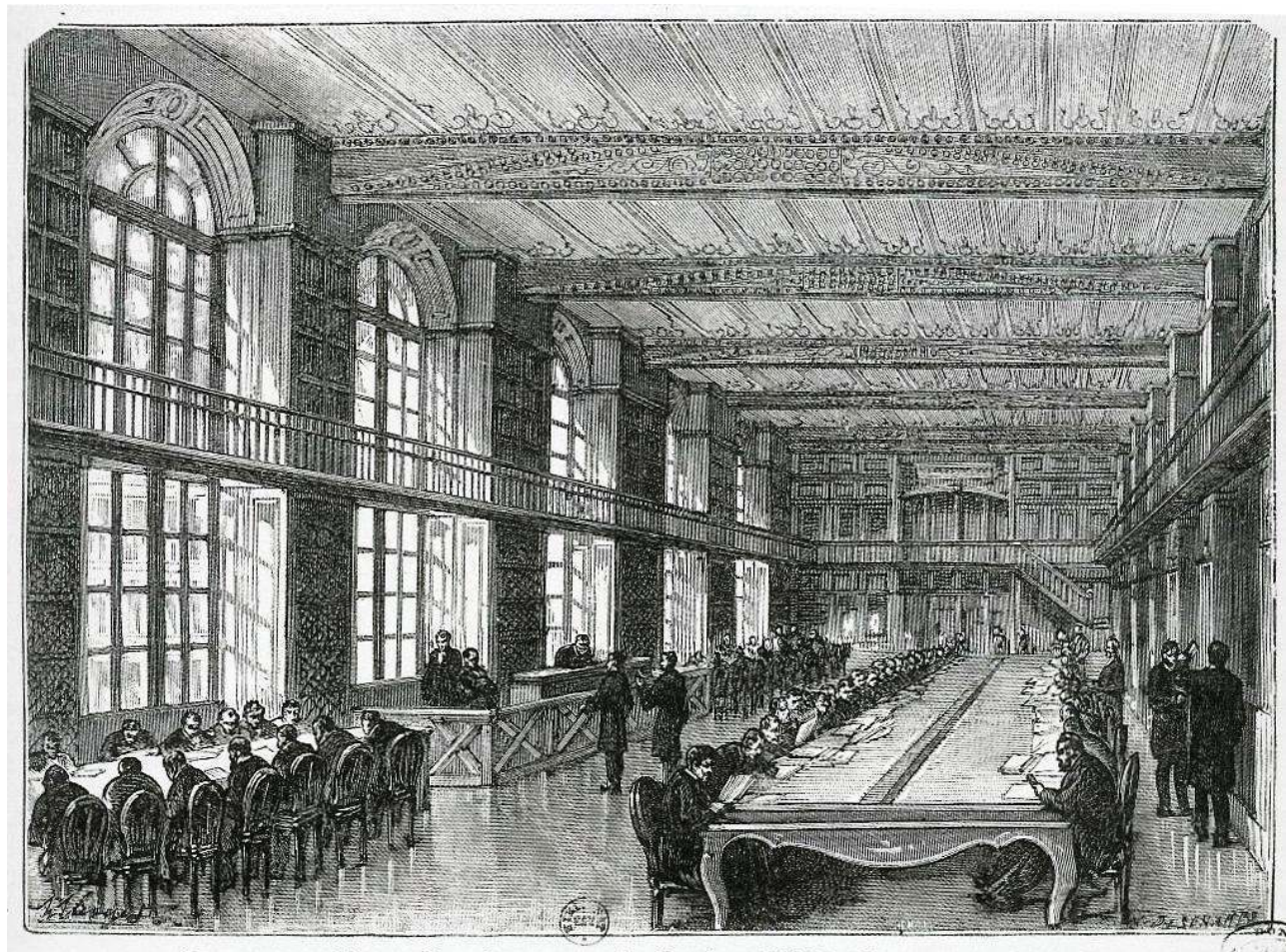

Jean-Louis Pascal

Dominique Varry (dir.), Histoire des bibliothèques françaises. Les bibliothèques de la Révolution et du XIXe siècle : 1789-1914, Paris, éd. du Cercle de la Librairie, 1991, p. 155

Considérée comme provisoire, elle était déjà trop exiguë lors de son ouverture car elle ne permettait d'accueillir qu'une centaine de personnes ${ }^{39}$ et vingt-cinq mille volumes. On y accédait de l'extérieur par la rue Colbert ${ }^{40}$, en traversant un premier salon destiné à la lecture de la presse qui n'offrait que huit places. Une soixantaine de périodiques y étaient représentés. Puis venait une galerie de trente mètres de long " admirablement éclairée » et « coquettement décorée »: «Les arcs surbaissés (ill. 6) qui marquent les différentes parties de la salle de lecture sont ornés de cartels décorés de feuillages de chêne, de laurier, de guirlandes de capucines ou de palmes au milieu desquelles figurent les noms d'imprimeurs et de bibliographes célèbres $»^{41}$. 
III. 6 : Division de travée de la nouvelle salle de lecture publique en vue d'une affectation au département des Manuscrits

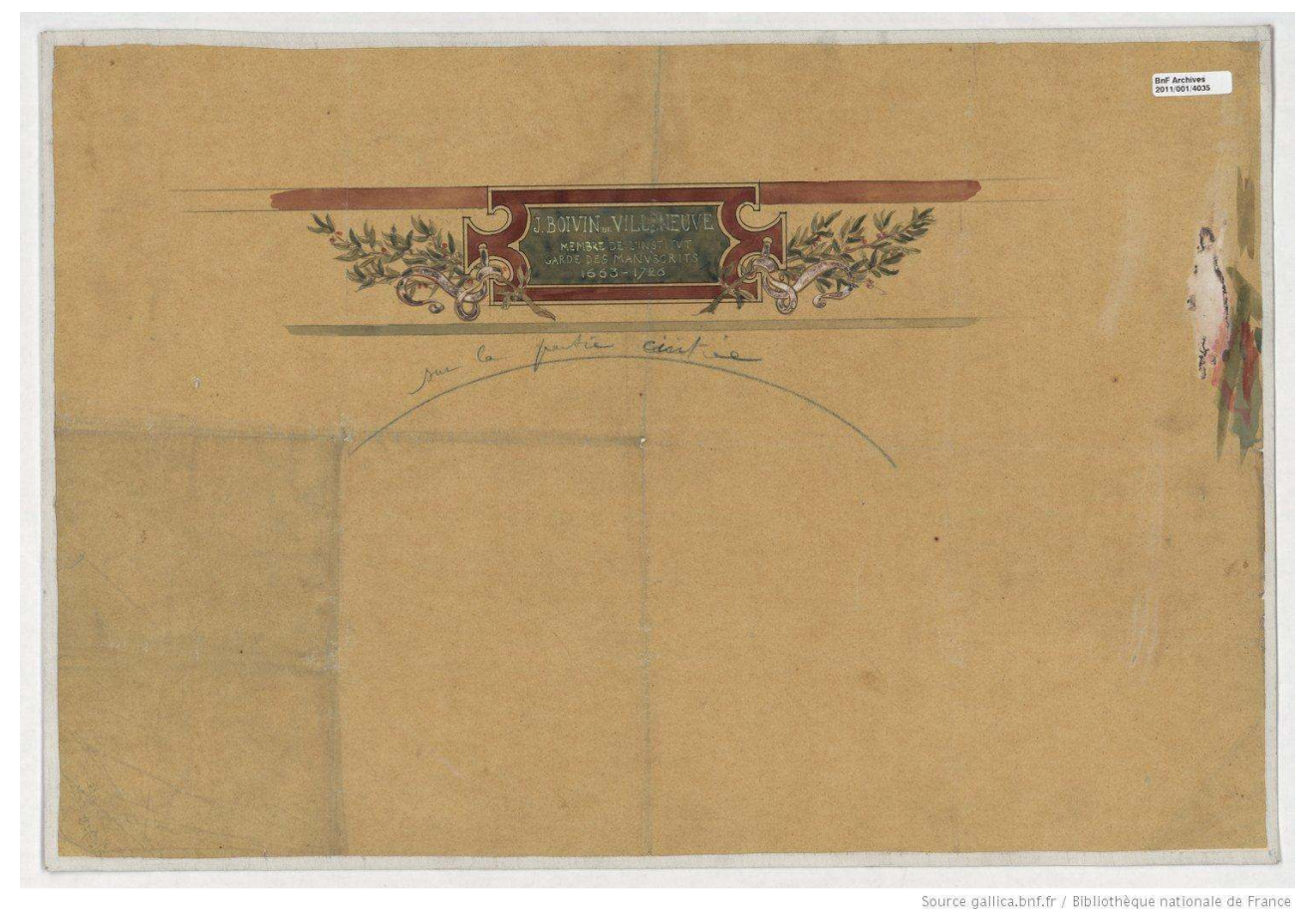

Jean-Louis Pascal. Avant 1881. Dessin aquarellé sur calque collé sur papier. 365 × $540 \mathrm{~mm}$

Arch. adm. B.n.F., 2011/001/4035

21 Pascal voulait que ces noms fussent en rapport avec la future affectation de la salle $e^{42}$ c'est-à-dire la réserve des manuscrits ; Léopold Delisle en dressa la liste suivante : Ulric Gering (1470-1509), Nicolas Jenson (1470-1481), Antoine Vérard (1485-1511), Les Estienne et les Didot ${ }^{43}$.

Les livres n'étaient pas en libre accès à la salle publique : « On a coupé la salle en hauteur par un balcon de fer, ce qui a permis d'installer deux services : en bas, la littérature ; sur l'étagère la théologie et la jurisprudence. Mais comme il restait deux services, [...] l'histoire et les sciences, il a fallu les loger dans les combles $»^{44}$.

La salle était équipée d'un système de transmission de bulletins et de deux montecharges, mais pas de l'électricité, malgré la demande que Pascal avait faite ${ }^{45}$. Titillé par le fait que le British Museum, le musée de South Kensington à Londres, la bibliothèque Sainte-Geneviève et la bibliothèque de l'École des beaux-arts de Paris étaient éclairés à l'électricité le soir, il demanda d'en essayer l'usage dans la salle de lecture provisoire rue Colbert et dans le comble :

«Je choisissais cette salle précisément parce qu'elle sera provisoire et qu'on y pourra, sans rien engager de définitif, faire les tâtonnements qui pourront conduire à la perfection. Hier, mon ami, M. Garnier, que j'interrogeais sur différents essais faits ou à faire à l'opéra, me disait qu'il comptait publier dans deux ou trois mois un rapport très circonstancié sur onze systèmes, pas tous électriques, qui lui avaient été proposés pour l'amélioration de l'éclairage de la scène et du grand foyer. $»^{46}$

En 1896, Pascal supprime l'entrée de la salle publique de lecture par le vestibule et l'escalier provisoire du $\mathrm{n}^{\circ} 3$ de la rue Colbert et il constitue une nouvelle entrée, à l'autre extrémité de la salle de lecture, pour préparer les travaux d'agrandissement de 
l'établissement ${ }^{47}:$ «On pourra entrer plus convenablement par l'autre extrémité de la salle de lecture en attendant la construction de la nouvelle salle $»^{48}$.

À cet effet, Pascal demande d'utiliser deux salles inoccupées de l'extrémité de l'aile gauche de la cour de l'administration pour y placer les volumes de la réserve : « Il y manque les installations intérieures pour y reporter les parties des collections dont le déplacement s'impose par suite du transfert de l'entrée provisoire de la salle Colbert dont les bâtiments d'accès, l'escalier en bois, les W.C., doivent faire place à des constructions définitives [...] On pourrait encore y abriter provisoirement la Bibliothèque Renan qui est restée dans les vieux locaux abandonnés de l'ancienne administration et pour une partie sur le sol [...] Si je ne puis les déplacer d'avance, il me sera impossible de reporter l'entrée de la salle publique $»^{49}$.

Tant bien que mal ${ }^{50}$, et malgré l'occupation de bientôt la moitié de ses locaux pour abriter les magasins de manuscrits, cette salle de lecture fonctionnera jusqu'au $1^{\mathrm{er}}$ janvier 1935. Le comble, qui sera entièrement semblable à ceux des bâtiments donnant sur la rue de Richelieu, deviendra un magasin pour les imprimés; les bureaux des acquisitions et du dépôt légal s'étendront dans les pièces du rez-de-chaussée.

\section{L'aile est de la cour d'honneur (1885) : la reprise d'un schéma existant}

27 La cour d'honneur était bordée dans toute sa longueur à l'est par un corps de bâtiment construit par Claude Armand Mollet pour John Law et transformé par Robert de Cotte en bibliothèque ${ }^{51}$. C'était le seul qui restait complet de l'ancienne bibliothèque du Roi. À l'extérieur, Pascal renouvelle tout le bâtiment en lui conservant autant que possible son caractère : «C'est à titre de spécimen de l'ancienne bibliothèque du roi qu'il m'a paru intéressant de reconstituer tout le premier étage scrupuleusement, dans lequel on installera la salle de lecture des manuscrits $\|^{52}$. Le fronton, jadis orné des armes de Louis $\mathrm{XV}$ reçoit un bas-relief traité dans le goût du XVIII ${ }^{e}$ siècle par le sculpteur Charles Degeorge, représentant La Science servie par des génies ${ }^{53}$.

Pascal était, à la différence de Labrouste, un amoureux du XVIII e siècle. C'est lui qui sera, avec son ami Julien Guadet (1834-1908), à l'origine de la réédition du «Blondel». Labrouste était un "progressiste», il "n'avait pas été nommé [à la Bibliothèque nationale] pour "repriser" des vieilleries, mais - il le prétendait - seulement pour la confection de choses nouvelles au goût du jour ${ }^{54}$. Dans un rapport fait par l'inspecteur général des bâtiments civils, Jules Andrée ${ }^{55}$, au conseil général des bâtiments civils ${ }^{56}$, on lit : « J'ai l'honneur de proposer l'adoption du projet de M. Pascal, le louant d'autre part du soin pieux avec lequel il restaure dans le caractère du temps ce qui reste de l'ancienne bibliothèque du Roi, vestiges précieux d'un art souple et charmant bien français qu'on tenterait de faire disparaître au nom d'un purisme douteux ${ }^{57}$ dont on peut louer les efforts, mais qui en est encore à faire ses preuves de vitalité ». En effet, l'œuvre de Labrouste à la Bibliothèque nationale avait été diversement appréciée :

«Ce fut l'époque d'une effrayante hécatombe. Tapisseries, meubles, tableaux, glaces disparurent à jamais, furent jetés ou vendus, brisés ou entassés dans les caves [...] Depuis Labrouste, il s'est produit un singulier revirement des idées. L'architecture de de Cotte longtemps méprisée est redevenue de mode avec tout ce qui, de près ou de loin, touche à l'art du XVIII e siècle [...] Qui sait d'ailleurs si, le siècle révolu, les bizarres et lourdes inventions installées par lui dans ces monuments ne 
bénéficieront pas à leur tour de la mode posthume, et si, dans la suite, les rondelles de charcuterie du vestibule ne seront pas prisées à l'égal des corps de bibliothèque de de Cotte? $»^{58}$

Jean-Louis Pascal était respectueux de l'architecture des siècles passés, aussi bien

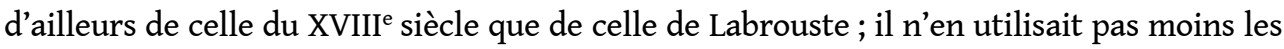
matériaux nouveaux dans ses restaurations. Il fit changer le comble en bois par un comble en fer ${ }^{59}$, pour abriter des magasins de livres et substitua aux planchers de bois, des planchers de fer ${ }^{60}$. Dans une lettre au ministre des travaux publics, datée du 20 février 1881, Pascal expose très précisément le travail qu'il réalise dans le bâtiment du fond de la cour d'honneur :

« Pour le bâtiment du fond de la cour, les opérations effectuées sont les suivantes: après avoir étrésillonné les baies du rez-de-chaussée (ill. 7), j’ai procédé à l'enlèvement des terres qui remplissent les intervalles entre les murs de fondation, à la création de soupiraux sur la grande cour et à l'établissement d'éperons intérieurs destinés à porter les poutres et le solivage du plancher bas du rez-dechaussée et j'ai pu ainsi constituer les caves qui manquent. Les prises d'air pour les calorifères ont été créées et les appareils eux-mêmes sont en cours d'exécution en ce moment. Un escalier nouveau met en communication le sous-sol et le rez-dechaussée et un autre escalier en fer et ardoise continue jusqu'au comble. Pendant la durée de ce travail le rez-de-chaussée était évacué mais le $1^{\text {er }}$ étage recevait encore plusieurs centaines de lecteurs tous les jours et le service ne pouvait y être interrompu un seul instant, non plus que pendant une seconde opération maintenant terminée, bien plus délicate que la précédente. Il s'agissait de découvrir et de recouvrir le bâtiment entier. Avant d'enlever la charpente en bois, un grand abri provisoire en charpente, couvert en zinc, a été élevé par-dessus le tout pour l'abriter. Ardoises, plombs, voliges, chevrons, arbalétriers, ont été démontés et descendus et les entraits en bois qui forment poutres pour l'ancien plancher ont pu être remplacés par des poutres en fer destinées à porter le solivage en fer, sans aucun accident et sans mettre à découvert la salle au-dessous. [...] La charpente en fer a pu être montée aussitôt, des chaînages établis. Les précautions les plus minutieuses ont été prises pour ne livrer aucun élément à l'incendie qui pourrait se propager du dehors en n'employant que du fer, du métal, du plâtre, de la terre cuite et de l'ardoise dans toute cette toiture, actuellement au trois quart terminée. La menuiserie intérieure des combles est fort avancée à l'atelier, ainsi que la serrurerie et les plaques de fonte, en sorte qu'aussitôt qu'il m'aura été possible de faire crever et remplacer le plancher haut en bois du $1^{\text {er }}$ étage, on pourra commencer à procéder à la pose de tout le mobilier intérieur préparé d'avance. La salle du $1^{\text {er }}$ étage est maintenant évacuée; je fais procéder au relevé des fragments d'architecture et de sculpture à restaurer et dans peu de semaines on aura pu commencer la substitution du solivage en fer au solivage en bois au plancher supérieur pour continuer ensuite une substitution analogue au plancher inférieur. » 61 


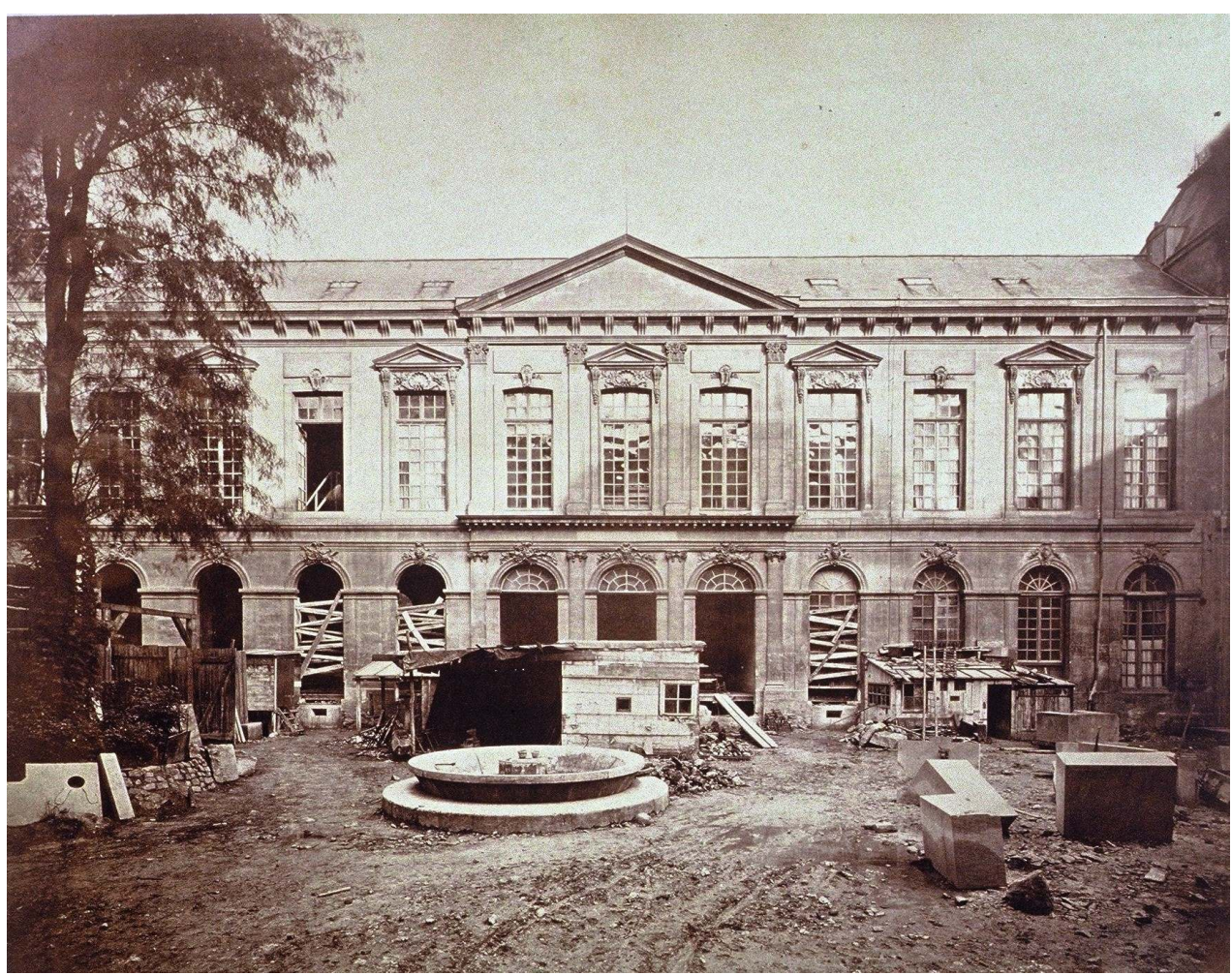

Aile est en cours de restauration, au début des années 1880. Jean-Louis Pascal, architecte

Archives nationales, VA album CX $n^{\circ} 15$ : Travaux à la Bibliothèque nationale

Les nombreuses parties de façade et les sculptures dégradées ou remplacées par du plâtre furent réparées soit en pierre soit en ciment métallique ${ }^{62}$, les soubassements furent refaits et toutes les menuiseries des fenêtres changées ${ }^{63}$ :

« Le soubassement du vieux bâtiment est en moellons et en moellons salpêtrés. Il faut que je raccorde cette surface avec une partie de pierre percée de soupiraux et que j'obtienne l'apparence de la pierre comme dans tout le reste de la cour. Cet essai a été tenté il y a deux ans. Depuis cette époque le revêtement testé au contact avec le sol même, n'a absolument pas bougé ; l'illusion est presque complète : il faut être prévenu pour s'apercevoir qu'il n'y a pas de pierre à cet endroit [...] Pour le reste de la façade, j'ai en maints endroits à substituer à des fragments d'enduit en plâtre ou en ciment, à des petits morceaux de pierre, des surfaces devant bien se raccorder avec le ton de la pierre et la nature du grain $»^{64}$.

Pour réussir ce raccord parfait, Jean-Louis Pascal prône un usage modéré du ciment métallique :

«Cette matière hygrométrique prend une autre couleur que la pierre; ceux qui l'emploient élargissent les joints et en font des rubans affreux sur l'appareil [...] On ne devra guère en faire l'emploi que dans les parties où l'on n'en pourrait ainsi dire pas constater la présence parce que la saleté, la suie, la poussière y auraient laissé des traces noires - pour les filets, les listels, les arêtes de larmiers, les dessous des corniches et des bandeaux - Il serait puéril en effet de ne pas tenir compte de l'économie que peut représenter l'emploi de ce ciment métallique appliqué en petites quantités, et très résistant, très adhérent, parfait à ce point de vue, quand on n'en fait pas des sortes d'enduits ${ }^{65}$. 


\section{Création d'un vestibule d'honneur au rez-de-chaussée de l'aile est} (ill. 8)

\section{8 : Le vestibule de l'administration}

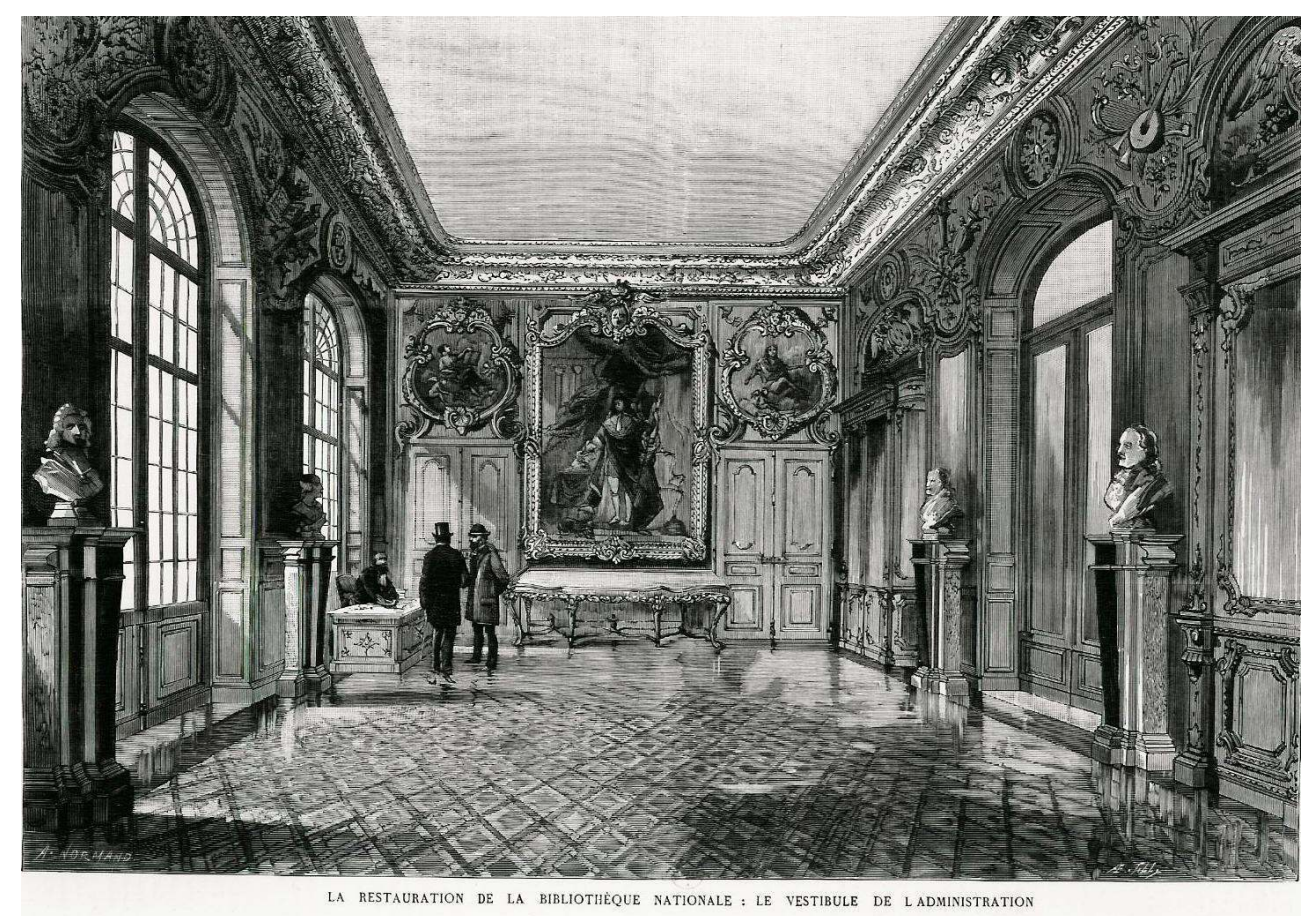

Rez-de-chaussée, aile est de la cour d'honneur de la Bibliothèque nationale. Restauration de JeanLouis Pascal

B.n.F., département des estampes Ha 132, cl. no 2000 C 230713

Pascal reconstruit complètement la première travée de l'aile est, jouxtant l'aile donnant sur la rue Colbert, y compris l'escalier en ardoise ${ }^{71}$ et y installe le cabinet du conservateur. Puis vient la salle du conservatoire " très sobre et seulement boisée dans le style voulu ». Pascal aménage ensuite au rez-de-chaussée de l'aile rénovée le bureau de l'administrateur et son vestibule : « Nous avons des tableaux de Boucher, Natoire, Van Loo, des cadres en bois sculpté, des moulures dorées provenant de l'ancien cabinet des médailles détruit lors 
de la suppression de l'arcade Colbert. J'en ferai l'emploi dans la salle centrale du rez-dechaussée qui deviendra le vestibule de l'administration $»^{72}$. Pascal fait restaurer les deux portraits de Louis XIV et Louis XV, dans leurs cadres dorés aux armes de France, qui se trouvaient avant la Révolution au milieu des extrémités du cabinet des médailles, dans la salle qui surmontait l'arcade Colbert. L'original du portrait de Louis XIV par Hyacinthe Rigaud (1659-1743) était perdu pour la Bibliothèque mais une copie en avait été faite sous la Restauration par le peintre Pierre Edme Louis Pellier, et lors de la destruction du cabinet des médailles, c'est cette toile qui y figurait. Pascal demande qu'une copie en soit réalisée par le peintre Joseph Fortuné Séraphin Layraud (1833-1913), ancien pensionnaire de Rome. « Il serait tout naturel, historiquement et archéologiquement, que le portrait de Louis XV revienne prendre sa place en face de celui de Louis XIV dans les conditions où il était primitivement $»^{73}$. Le portrait de Louis XV par Louis Michel Van Loo (1707-1771) avait été remplacé sous la Restauration par un portrait de Louis XVIII attribué à Ary Scheffer (1795-1858) $)^{74}$; «Je crois que nous avons encore le portrait original de Louis XV, abîmé, détérioré, fendillé, mais peut-être restaurable $»^{75}$; Pascal pense que ce portrait est dû à Van Loo: "dans le grand vestibule d'honneur précédant le cabinet de $\mathrm{M}$. l'Administrateur, vestibule inachevé [...il y a ] quatre magnifiques Boucher et un original de Van Loo $»^{76}$, portrait qui aurait été commandé pour le cabinet des médailles en même temps qu'on faisait faire les trumeaux par Charles Joseph Natoire (1700-1777) et Carle Van Loo (1705-1765) et les quatre dessus-de-porte représentant des muses par François Boucher (1703-1770).

Pascal demande que ce soit aussi Joseph Layraud qui le restaure : «Je voudrais que le peintre désigné plus haut fût chargé de se servir de ce qu'on donne sur notre toile pour en faire un digne pendant de la copie de Rigaud, et les éléments d'une restitution y sont certainement suffisants ${ }^{77}$. Henri Bouchot, conservateur à la Bibliothèque nationale dans les années 1890, nous donne la description de ce vestibule :

"C'est dans le vestibule de de Cotte, repris pièce à pièce, reconstitué tout entier, que sont aujourd'hui placées les quatre toiles de Boucher arrachées au cabinet des médailles. Les voici dans leurs cadres chantournés, décorés de coquilles et de masques, tous fraîchement redorés, comme on les voyait autrefois. Au milieu et se faisant vis-à-vis, dans leurs encadrements anciens, Louis XIV et Louis XV, en grand costume, en leur qualité de fondateurs de la maison. Puis, aux moulures des plafonds, les attributs d'art et de littérature projetés par de Cotte pour les salles de la Bibliothèque $»^{78}$.

Pascal décore en effet quatre dessus-de-porte du vestibule grâce aux Muses de François Boucher et il fait sculpter par Gustave Germain, d'après des moulages réalisés dans ce qui reste de l'ancien cabinet des médailles ${ }^{79}$, dans l'hôtel Colbert (au n ${ }^{\circ} 12$ de la rue Colbert), des trophées représentant La Géographie, La Marine (ill. 9), La Science, La Musique, L'Agriculture et $L a$ Guerre, dont il orne les lambris hauts du vestibule 


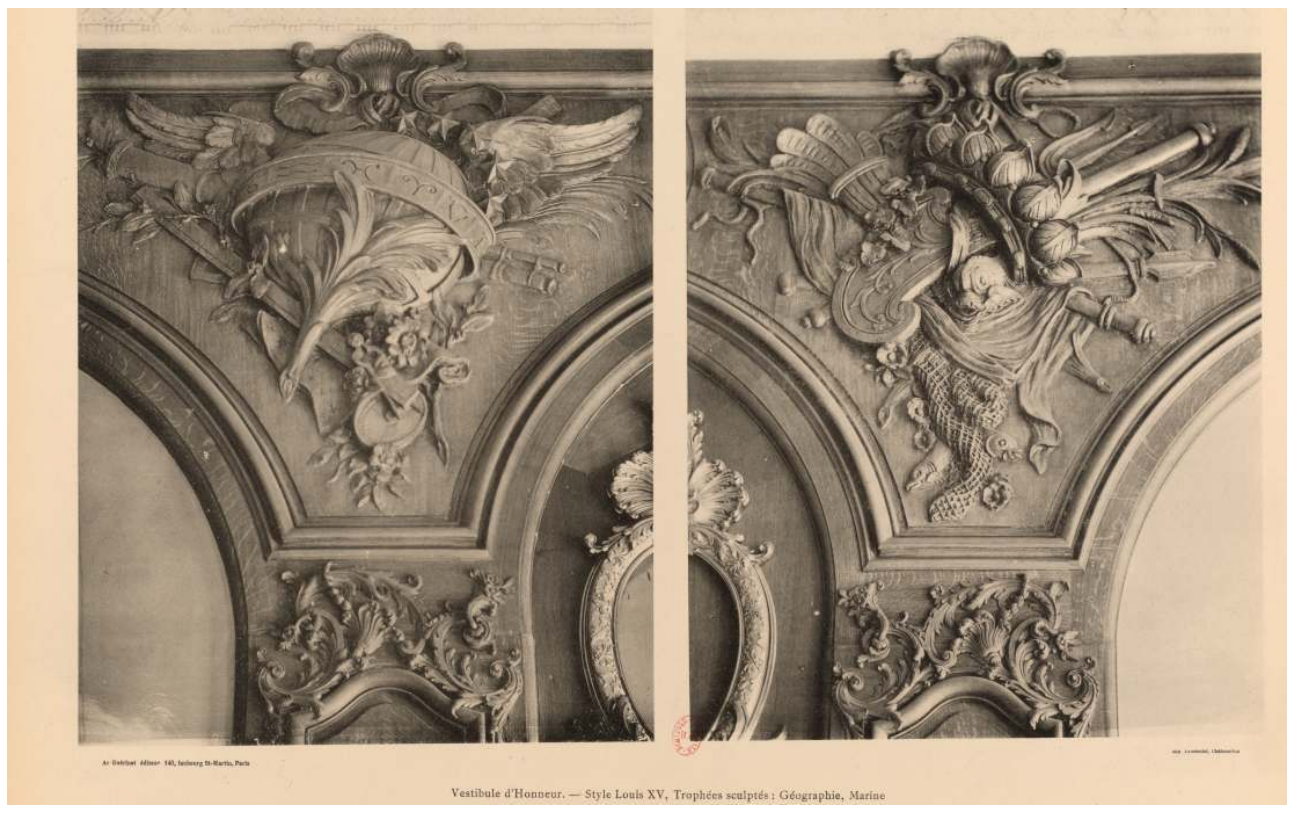

Style Louis XV, trophées sculptés : Géographie, Marine. Reconstitution par l'architecte J.-L. Pascal et le sculpteur Gustave Germain

Arch. adm. B.n.F., 2007/061/001, pl. 175-176

Le marbrier Drouet Langlois est choisi pour exécuter le dallage du vestibule pour la somme de huit mille francs ${ }^{80}$. Le vestibule est orné de quatre bustes du duc de Berry (1340-1416), frère de Charles V, d'Henri Estienne (1528-1598), de Gaston d'Orléans ${ }^{81}$ (1608-1660) et de Daniel Huet ${ }^{82}(1630-1721)$, évêque d'Avranches, bustes posés sur des gaines de brèche violette de Serravezza ${ }^{83}$. Le centre de la pièce est orné en 1907 d'une baigneuse $^{84}$ de marbre de François Léon Sicard (1862-1934). Pascal voulait que le vestibule de l'administration servît de salle d'exposition des acquisitions récentes de la Bibliothèque et il demandera, jusqu'à son départ de la Bibliothèque, la confection d'un mobilier adapté ${ }^{85}$. Des tapisseries décoratives en camaïeu sur fond rouge sont commandées, d'après des cartons de Lavastre et Luc-Olivier Merson (1846-1920), exécutées en Savonnerie par la manufacture des Gobelins ; ce sont : La Poésie, La Science et La Mécanique ${ }^{86}$. C'est le futur administrateur général Henry Marcel (1854-1926) qui propose à Pascal, pour exécuter les bordures de ces panneaux de tapisserie qui viendront décorer l'antichambre du cabinet de l'administrateur, le jeune décorateur Lavastre dont il vient de voir, au théâtre de Rouen, un rideau dans le style de Jean Berain (1637-1711), «d'une composition et de colorations charmantes». Marcel pense néanmoins que «les motifs décoratifs qui s'accorderaient le mieux avec le style des boiseries que rétablit [Pascal] dans la pièce dont il s'agit devraient être quelque chose d'analogue aux feuilles de paravent composées par Watteau et gravées par L. Crépy. Les motifs de Berain, d'époque antérieure, s'accorderaient mieux avec des boiseries du temps de Louis XIV ${ }^{87}$.

Puis vient le cabinet de l'administrateur, avec son mobilier créé par Pascal d'après de vieux modèles : porte-atlas, tables, chaises, fauteuils, cheminée, corps de bibliothèque de de Cotte; Pascal commande de luxueux cartonniers, pour voisiner avec les boiseries sculptées. L'ameublement est complété par un beau bureau Régence à cuivres ciselés et un splendide cartel en émail vert ${ }^{88}$, pendule du XVIII siècle qu'il se procure chez 
l'horloger Boure, 5 rue de l'Entrepôt, à Paris ${ }^{89}$. Il fait faire des rideaux sur dessins spéciaux, achète tapis ${ }^{90}$, et chenets pour la cheminée sculptée.

À droite du vestibule d'honneur sont situés les bureaux de l'administration boisés de chêne, meublés également dans le style. Dans la pièce d'entrée et les couloirs des bureaux de l'administration fréquentés par le public, Pascal installe à la demande de Delisle, une cinquantaine de portraits gravés, dans des cadres dorés, doubles du département des estampes, sorte de musée des bienfaiteurs et fondateurs des collections de la Bibliothèque ${ }^{91}$ : «Il faut regretter que le couloir ait subi l'influence de M. Labrouste; ses meubles en bois à bandeaux noirs semblent, dans ce merveilleux décor environnant, un meuble de salle à manger bourgeoise ${ }{ }^{92}$.

\section{Création d'une salle de lecture des manuscrits au $1^{\text {er }}$ étage de l'aile est (1886)}

Pascal installe à l'étage ${ }^{93}$ l'actuelle salle de travail du département des manuscrits (ill. 10) ouverte au public le 18 octobre $1886^{94}$.

\section{10 : Salle de lecture du département des manuscrits}

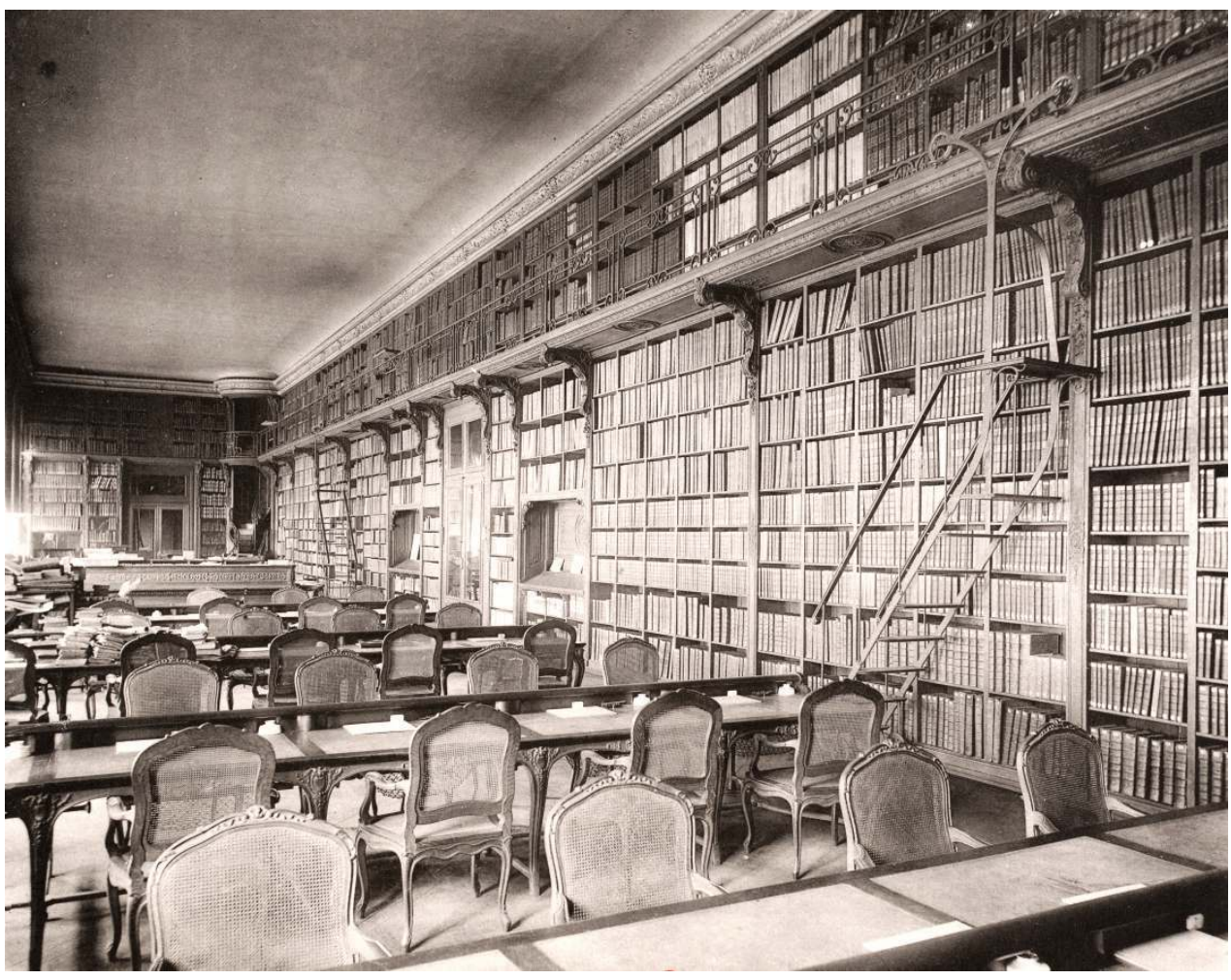

Reconstitution avec des éléments d'époque Louis XV, par l'architecte J.-L. Pascal et le sculpteur Gustave Germain

Arch. adm. B.n.F., 2007/061/001, pl. 233

41 Là aussi il adopte un parti différent de son prédécesseur : il réutilise les anciennes boiseries, restitue les boiseries délicates du temps de Louis XV en faisant exécuter les copies nécessaires pour assurer la liaison : «Cette salle des manuscrits est restée d'aspect telle que Robert de Cotte l'avait aménagée pour les livres imprimés; elle a ses mêmes 
corps de bibliothèque, toute son admirable décoration sur bois d'avant ${ }^{95}$. Pascal parle de « restitution quasi archéologique»:

«Pour arriver à ce résultat, j'ai fait démonter et débarrasser des couches superposées de peinture qui les encrassaient, les beaux spécimens de menuiserie décorée, du siècle dernier, qui existaient dans la grande salle du premier étage ; j'ai fait conserver les vieux bois provenant des planchers démolis pour compléter ce qui devait être fait dans cette salle ; enfin j'ai réuni tout ce qui restait encore dans divers endroits, notamment dans mon agence, des boiseries sculptées de l'ancien cabinet des médailles. Ces débris d'un art intéressant, abandonnés et quasi perdus, ont besoin de grandes réparations ; il faut d'autre part constituer pour les raccorder beaucoup de parties neuves afin de les adapter aux formes et aux dimensions des salles auxquelles elles n'avaient pas été destinées [...] Ce sculpteur [Germain] a déjà fait à mon entière satisfaction la restitution des sculptures de la façade intérieure du bâtiment sur la rue Colbert $»^{96}$.

Gustave Germain réalise le travail de restauration de sculpture sur bois de l'intérieur du bâtiment est de la cour d'honneur ${ }^{97}$. Il restaure notamment la corniche Louis XV de la grande salle du premier étage ${ }^{98}$. Gustave Germain travaillait en même temps pour le duc d'Aumale à Chantilly ${ }^{99}$ et y restaurait de vieilles boiseries du XVIII ${ }^{\mathrm{e}}$ siècle : "Sa compétence et son habileté pour les restaurations de cette époque m'ont longtemps fait désirer [dit Pascal] de laisser la même main terminer tous ces travaux; actuellement encore, il exécute pour le duc d'Aumale à Chantilly un travail absolument semblable de restauration de vieilles boiseries du siècle dernier et j'ai pu m'assurer par les visites fréquentes que je fais à son atelier qu'on ne saurait y apporter plus d'adresse et plus d'habileté $»^{100}$. Pascal le tenait en haute estime : «M. Germain, auteur de la plupart des sculptures de la Bibliothèque nationale, sculpteur que j'ai employé depuis de nombres d'années dans des travaux de toutes sortes, à ma grande satisfaction " ${ }^{101}$. Auguste Ronsin, spécialisé dans la menuiserie d'art et de bâtiment, réalise également des boiseries au rezde-chaussée ${ }^{102}$. Le menuisier Mathérion réalise toute la menuiserie de la salle de de travail des manuscrits, y compris la construction des deux escaliers ${ }^{103}$. La restauration du parquet en feuilles de la future salle des manuscrits fait l'objet de ce que l'on qualifierait de nos jours de restauration « à l'identique »:

«Tout doit être désassemblé, déchevillé avec précaution pour ne pas endommager les bois dont la conservation importe au ton et à l'ensemble de la salle. Tous les encadrements de panneaux devront être tirés de large et les rainures ou languettes refaites. Les panneaux fendus seront à remplacer avec les vieux bois que l'on refend en ce moment à la bibliothèque, dans de vieilles poutres. [...] Toutes les feuilles devront être transportées à l'atelier pour y être réparées [...] Il a fallu dix-sept heures pour désassembler, réparer et remettre en état d'être reposées les feuilles emportées [...] Les encadrements des feuilles sont également refaits au moyen des vieux bois de l'administration. Enfin la grande frise d'encadrement du pourtour de la salle, est faite aussi en vieux bois et disposée suivant les nécessités des compartiments. $»^{104}$

Pascal refusera la pose d'un linoléum dans la salle de travail des manuscrits, demandée par le conservateur et le public pour atténuer les bruits, parce que le plancher présente un intérêt artistique ${ }^{105}$. Léon Labrouste n'était pas tendre avec l'œuvre de Robert de Cotte à la Bibliothèque nationale, peut-être par atavisme ; parlant de la façade du bâtiment du fond de la cour d'honneur, il écrit :

«Au premier étage [...] l'ordre corinthien est maigre, son chapiteau est raide, sa composition sèche, son tailloir pauvre et écrasé sous l'énorme entablement qui le surmonte où des consoles, absolument disproportionnées, soutiennent une 
corniche sans larmier. Les lourdes consoles, qui reposent sur une architrave très mince et décorent une haute frise, sont de l'effet le plus malheureux $»^{106}$.

Il avait en revanche une haute opinion de la restauration que Pascal avait menée dans le bâtiment du fond de la cour d'honneur, peut-être parce que ce dernier avait respecté l'œuvre de son père à la Bibliothèque nationale :

«La décoration intérieure du premier étage présente un ensemble remarquable de sobre richesse et de distinction. Impossible de trouver des boiseries plus délicatement sculptées comme détails et plus belles d'ensemble par leur gracieuse fermeté. C'est dans cette décoration, dans cet aménagement, dans le parti adopté par l'architecte, dans les dispositions pour remplir un programme et le satisfaire qu'il faut admirer l'œuvre de Robert de Cotte. On trouve dans les moindres parties de ce vaste tout une unité de vue, qui n'a pas empêché l'originalité des artistes qui concouraient au travail général, de se produire, tout en restant à l'unisson dans leur individualité. Ces boiseries (ill. 11) se composent de montants de casiers à livres ornés de rinceaux et de rosaces terminés par de fins feuillages admirablement sortis de la masse du bois. [...] Les consoles en bois de la galerie sont d'un travail remarquable, les chutes de fleurs qui les terminent sont d'une exécution et d'une composition qui ne laisse rien à désirer (ill. 12). La muraille du côté de la cour avait reçu au commencement de ce siècle des casiers d'un style bien différent de ceux du reste de la salle, ils ont été démolis et derrière, on a retrouvé les cadres d'anciennes peintures qui avaient été enlevées lors de l'installation des casiers qui les auraient masquées. Aujourd'hui, on a restauré d'anciennes peintures de Van Loo et de Natoire [...] Toute cette décoration venait de l'hôtel de Mme de Lambert [...] Les cadres avaient été mutilés, les chiffres, les couronnes et les fleurs de lys enlevés ; mais le tout a été habilement restauré par l'éminent l'architecte Jean-Louis Pascal qui a conduit d'une manière remarquable cet intéressant travail. $»^{107}$

III. 11 : Détails d'ornements
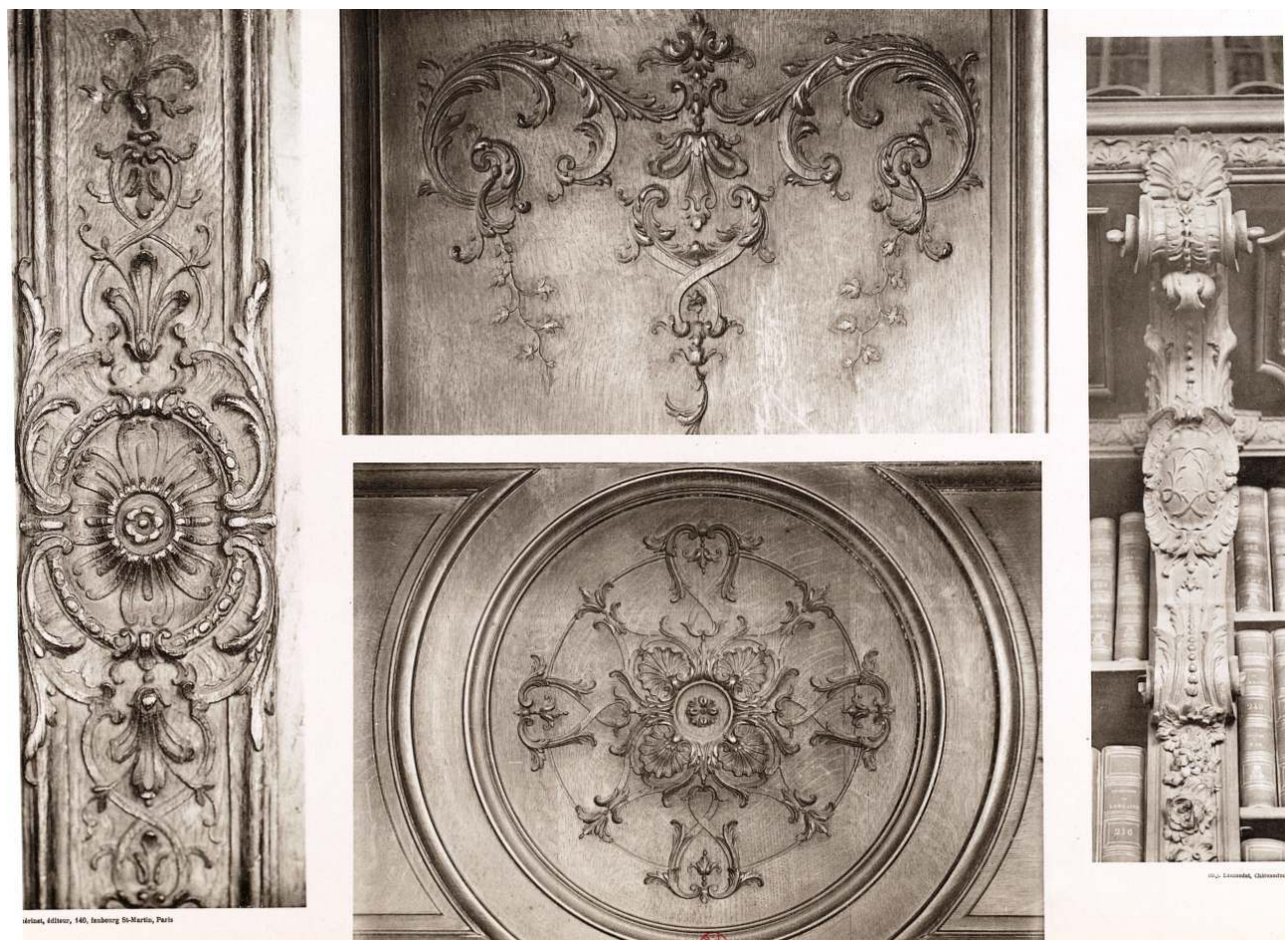

Consoles de style Louis XV. Salle des manuscrits. Sculpteur Gustave Germain et menuisier Mathérion

ARCH. ADM. B.N.F., 2007/061/001, PL. 236 À 239. 


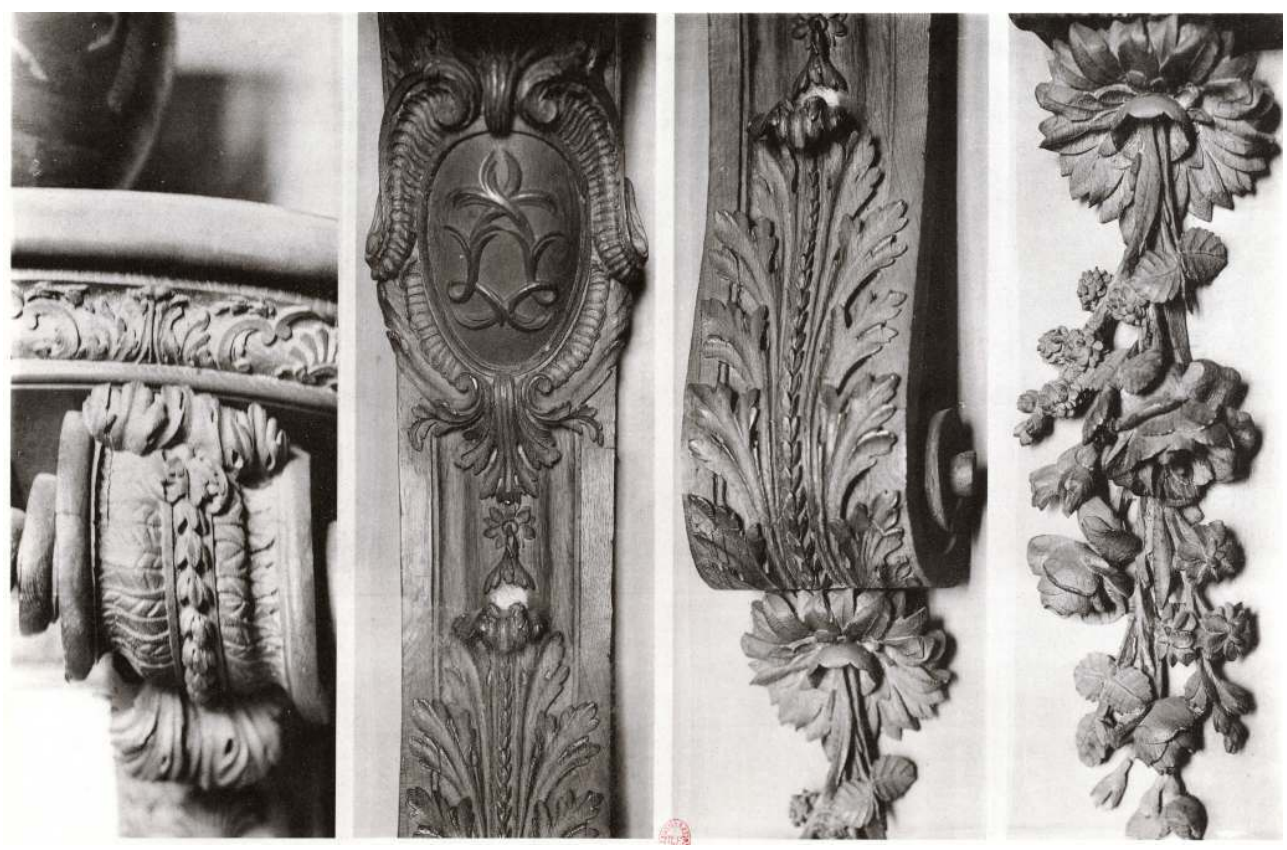

Détails de consoles Louis XV et Louis XVI. Salle des manuscrits. Sculpteur Gustave Germain et menuisier Mathérion

Arch. adm. B.n.F., 2007/061/001, pl. 240 à 243

Pascal a voulu que les lecteurs ne soient pas obligés de se rendre à la salle des imprimés lorsqu'ils voulaient vérifier un texte sur celui d'un manuscrit; des livres imprimés occupent cinq des rayons de la salle nouvelle. Quant aux manuscrits, ils ne sont, sur cent mille que possède la Bibliothèque, qu'au nombre de dix mille environ sur les rayons de cette salle. Les quatre-vingt-dix mille autres sont placés dans les combles, que l'architecte a mis en communication avec la salle de lecture des manuscrits par deux monte-charges et toute une installation de sonneries et de timbres avertisseurs qui assurent la rapidité du service ${ }^{108}$. Le mobilier de la salle des manuscrits est composé de dix grandes tables à dix places, on peut y feuilleter à l'aise les volumes les plus encombrants, une table à quatre places, une à trois places, deux tables pour les garçons de bureau, et cent sièges. Le choix du mobilier fait l'objet d'une discussion entre Théodore Mortreuil, secrétaire général de la Bibliothèque nationale et Pascal :

«On peut diminuer la largeur de la table, si c'est utile. La mesure de la profondeur du modèle est bonne. La peau de porc devrait être remplacée par une simple teinte en noir. Pour les encriers et les plumiers, il y aurait à disposer dans la table, à droite et à portée des lecteurs, une entaille en creux qu'on recouvrirait d'une coulisse au niveau de la table. Le champignon en bois pour suspendre le chapeau et le filet qui sont fixés aux tables actuelles du département des manuscrits sont très utiles. Il faudrait en munir les tables nouvelles. Pour les fauteuils, le cannage semble bien préférable à la peau de porc. $»^{109}$

Pascal avait commandé à l'entreprise Quignon les chaises et fauteuils de la nouvelle salle de lecture de l'aile Colbert, et ceux-ci s'étaient rapidement détériorés. Pascal propose de changer de fournisseur en vue de la commande pour la nouvelle salle des manuscrits. Il avait remarqué le travail d'un ébéniste marseillais, Auguste Blanqui ${ }^{110}:$ «L'exposition des arts décoratifs m'avait donné une occasion de remarquer des meubles très soigneusement traités par un ébéniste marseillais, M. Blanqui ». Pascal propose de mettre l'ébéniste 
marseillais en concurrence ${ }^{111}$ avec les grands ébénistes parisiens: G. Quignon "qui a exécuté déjà beaucoup de sièges pour la bibliothèque, d'excellents autrefois, de beaucoup moins bons dans sa dernière fourniture ", Fourdinois ${ }^{112}$ "dont la réputation est une garantie exceptionnelle », Godin, « fournisseur habituel de grandes administrations, et un spécialiste M. Redond ${ }^{113}$, fabricant de chaises, dont je ne suis pas encore assuré qu'il puisse présenter les garanties désirables pour les tables "; Pascal fournit les modèles à Blanqui :

«Je lui confiais ces modèles, sur l'ébénisterie desquels je n'aurais rien à dire si la sculpture était à la hauteur de l'ébénisterie. Mais il s'agissait de détails à traiter pour ainsi dire archéologiquement ; il n'y a pas lieu d'être surpris qu'il faille des retouches pour les harmoniser avec le style très accusé des ornements de l'architecte Robert de Cotte. Je me propose donc de faire exécuter sous mes yeux par M. Germain, chargé de tout le travail de sculpture de cette salle, les rectifications nécessaires. $»^{114}$

Fourdinois, sculpteur et tapissier, exécutera les tables en bois sculpté115 et c'est encore la maison Quignon qui fournira cent fauteuils de style Louis XV, en chêne, avec siège et dos canné ${ }^{116}$. Les tables, les fauteuils, le bureau des conservateurs ont été copiés sur ce qui restait de l'ancien mobilier: «La finesse de leur sculpture montre que, sur ce fait, les maîtres de l'œuvre moderne ne sont en rien inférieurs à leurs aînés; mis en regard, ils prêtent à la confusion complète, et témoignent du soin et de l'érudition du directeur des travaux $»^{117}$. Les tableaux de Charles Natoire et Carle Van Loo, enlevés à l'ancien cabinet des antiques, ont trouvé place dans les panneaux d'entre les croisées.

Outre le mobilier et la décoration des entre-fenêtres, Pascal devait placer, aux deux extrémités de la salle des escaliers en colimaçon moins rudes que les anciens, des pupitres dans les armoires mêmes, pour la consultation des catalogues, et un placard orné de glaces pour y resserrer les manuscrits les plus précieux. "Ici, encore, il s'est montré l'adaptateur savant, l'architecte érudit qui n'a point rêvé de faire mieux que de Cotte $»^{118}$. Toute la décoration de ces escaliers, véritables chefs-d'œuvre de menuiserie, est inspirée des doucines des balcons, des pilastres et des consoles de la salle dont l'architecte de Louis XV avait donné les profils; mais Pascal «a su aussi marquer d'une empreinte personnelle la construction des deux escaliers d'angle qui desservent l'étage supérieur ainsi que les galeries de la salle et dont le développement circulaire est tout à fait gracieux et original $»^{119}$. Peut-être en avait-il eu l'idée en visitant, lors de l'exposition universelle de Vienne en 1873, la Prunksaal de la Bibliothèque nationale autrichienne construite de 1723 à 1726 , au sein de la Hofburg, d'après les plans de l'architecte Johann Bernhard Emmanuel Fischer von Erlach (1656-1723) par son fils Joseph Emmanuel (1695-1742) pour l'empereur Charles VI (1685-1740). Aux angles de la salle, dont la forme rectangulaire s'hypertrophie au centre en un espace ovale destiné à magnifier la statue de l'empereur-mécène en Hercule ${ }^{120}$ sous un dôme ${ }^{121}$ décoré d'une fresque de Daniel Gran (1694-1757) en 1730, de beaux escaliers circulaires en bois permettent d'accéder à l'étage supérieur des armoires de livres en noyer.

49 Pascal, respectueux de ses prédécesseurs, a dû, pour ne pas faire jurer les styles entre eux, tenir compte du vestibule d'entrée imaginé par Labrouste. La porte du département des manuscrits (ill. 13), au premier étage, a été dessinée suivant les données néo-grecques du précédent ordonnateur : «C'est un chef d'œuvre de ciselure, et pourtant son apparente singularité déroute au milieu des œuvres de Robert de Cotte rencontrées à l'intérieur $~^{122}$. 

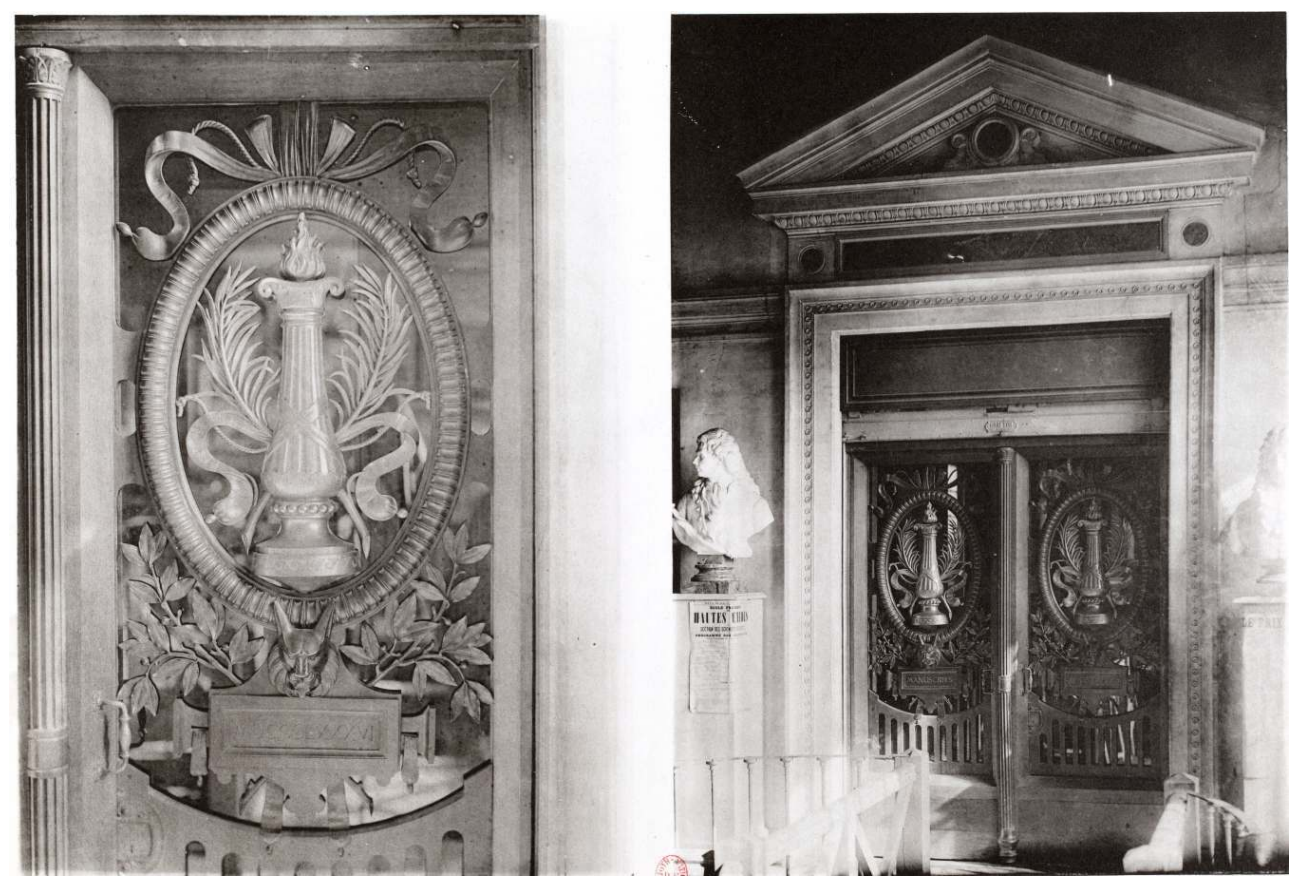

Jean-Louis Pascal. 1886. Cette porte de bronze ouvre sur le deuxième palier de l'escalier d'honneur de la bibliothèque nationale

ARCH. ADM. B.N.F., 2007/061/001, PL. 69

Les merveilleuses rampes des balcons, avec leurs soleils, leurs volutes, leurs tirebouchons, leurs grandes lettres « $\mathrm{L}$ » liées, que Robert de Cotte avaient projetées, ont été remplacées, au XVIII ${ }^{e}$ siècle, par des serrureries trop simples si l'on considère la richesse de la sculpture sur bois. Ce sont les mêmes que Pascal a gardées et copiées dans ses escaliers : « Ce qui est surprenant dans cette besogne un peu fastidieuse de reconstitution, c'est que même les objets de nécessité moderne soient de bonne allure entrés dans la disposition et l'ordonnance générales au point de ne se démêler pas du reste. Le tambour de la porte a son caractère ancien, les tableaux même sont absolument mariés au reste. De Cotte, revenant, aurait peine à s'imaginer qu'il n'est pas l'auteur de ces choses $»^{123}$.

Cette restitution « à l'identique » du bâtiment du fond de la cour d'honneur par Pascal fut unanimement louée : «Le manque de proportions idéales reproché par Blondel à de Cotte a perdu de sa valeur, eu égard aux tristes combinaisons mises en place par M. Labrouste. Le fond de la cour de cette bibliothèque est un palais, le devant est une gare de chemin de fer $\aleph^{124}$. Après ce travail de restauration, Pascal a désormais les mains libres; il peut maintenant faire œuvre personnelle dans l'angle nord-est de la Bibliothèque dont les propriétaires des maisons particulières qui subsistaient depuis le début du XVIII ${ }^{e}$ siècle sont expropriés en 1881. Pascal y réalise le « carré Vivienne » dont les bâtiments, servant d'écrin à la grande salle Ovale, vont accueillir le nouveau cabinet des médailles 


\section{NOTES}

1. Monique Eleb, Anne Debarre, L'Invention de l'habitation moderne, Paris, 1880-1914. Architectures de la vie privée, suite, Paris, Hazan et Archives d'Architecture Moderne, 1995, $534 \mathrm{p}$.

2. Jean-Louis Pascal est nommé architecte du diocèse de Valence (Drôme) le 30 mars 1875 en remplacement d'Antoine Nicolas Bailly (1811-1891). Arch. nat., $\mathrm{F}^{12}$ 5228, dossier de Légion d'honneur.

3. «Chaque fois qu'il y a moyen de rétablir sûrement ce qui a pu exister primitivement, j'ai tenu la main et je continuerai à exiger qu'on remplace les anciens matériaux abîmés par des matériaux de forme et de nature semblables à ceux qu'on déposera. » Arch. nat., $F$ ${ }^{19} 7914$, rapport de l'architecte diocésain sur les dépenses à faire en 1880, adressé au ministre par Pascal, 18 juin 1880.

4. Arch. nat., $\mathrm{F}^{21} 5855$, Bibliothèque nationale, grands travaux, rapport adressé par Pascal au ministre des travaux publics le 30 mai 1876.

5. Léon Labrouste, "Bibliothèque nationale à Paris. Bâtiments sur la cour d'honneur ", R.G.A.T.P., $\mathrm{n}^{\circ} \mathrm{XXXVI,} \mathrm{1879,} \mathrm{p.} \mathrm{3-4.}$

6. Léon Labrouste, La Bibliothèque nationale, son début et ses accroissements, ses bâtiments et ses constructions, ses agrandissements, ses travaux, Paris, H. Luthier, 1885, 94 p., p. 37.

7. Prédécesseur de Léopold Delisle au poste d'administrateur de la Bibliothèque (1858-1874).

8. Ève Netchine et Edmée Srauch, » La salle B », Mélanges autour de l'histoire des livres imprimés et périodiques, Bruno Blasselle et Laurent Portes (dir.), Paris, B.n.F., 1998, 369 p., p. 242-266.

9. Léon Labrouste, La Bibliothèque nationale, son début et ses accroissements, ses bâtiments et ses constructions, ses agrandissements, ses travaux, op. cit., p. 38.

10. Claire Lesage, » Henri Labrouste ", Mélanges autour de l'histoire des livres imprimés et périodiques, op. cit., p. 225.

11. Henri Bouchot, «Les derniers travaux de décoration exécutés à la Bibliothèque nationale par M. Pascal, architecte », Revue des arts décoratifs, $12^{\mathrm{e}}$ année, 1891-1892, p. 94.

12. Arch. nat., $\mathrm{F}^{21} 5855$, B.N., grands travaux, direction des bâtiments civils, travaux extraordinaires, 1877, lettre de Pascal du 24 décembre 1876 au ministre des travaux publics.

13. Ibid., B.N., grands travaux, 1877, supplément au rapport relatif à la reconstruction du bâtiment sur la rue Colbert, Jean-Louis Pascal, 12 janvier 1877.

14. L'aile est de la cour d'honneur.

15. Arch. nat., $\mathrm{F}^{21} 5855$, Bibliothèque nationale, grands travaux, 1877, supplément au rapport relatif à la reconstruction du bâtiment sur la rue Colbert, Jean-Louis Pascal, 12 janvier 1877.

16. Ibid. 
17. Ibid., conseil général des bâtiments civils, séance 7 août 1877. Rapport fait au conseil par M. Duc, Inspecteur général.

18. Léon Labrouste, La Bibliothèque nationale, son début et ses accroissements, ses bâtiments et ses constructions, ses agrandissements, ses travaux, op. cit., p. 37-38.

19. Arch. nat., $\mathrm{F}^{21} 5855$, B.N., grands travaux, 1878 , lettre de Pascal au ministre des beauxarts, 6 janvier 1879.

20. Henry Marcel, Henri Bouchot, Ernest Babelon, Paul Marchal, Camille Couderc, La Bibliothèque nationale, op. cit., p. 8.

21. Léon Labrouste, La Bibliothèque nationale, son début et ses accroissements, ses bâtiments et ses constructions, ses agrandissements, ses travaux, op. cit., p. 35.

22. Fils et élève d'Aimé Napoléon Perrey, et de Jouffroy, il entre à l'E.B.A. en 1868 ; auteur prolifique de statues de plâtre, statuettes en bronze, portraits en buste (Bazaine, 1870 ; Belgrand, 1879) et en médaillon, il sculpte en pierre pour l'église Saint-Eustache une Sainte Geneviève en 1873, Le génie de la chasse, groupe en pierre pour le nouveau Louvre, Le Génie civil, statue de pierre pour le palais du Trocadéro. Émile Bellier de la Chavignerie, Louis Auvray, Dictionnaire général des artistes de l'École française depuis l'origine des arts du dessin jusqu'à nos jours, architectes, peintres, sculpteurs, graveurs et lithographes, Paris, H. Loones, 1885, vol. 2, p. 240-241.

23. Gustave Germain avait notamment donné le modèle de la belle rampe du vestibule du Grand Château de Chantilly.

24. En 1878, Pascal s'embarque pour le Portugal qui l'a choisi comme architecte des galeries portugaises qui s'insèreront dans la grande rue des Nations du palais du Champ de Mars de l'exposition universelle de 1878. Il y fait reproduire par Watrinelle et Germain le porche de l'église du monastère des Hiéronymites de Belem près de Lisbonne et un cloître dont un premier groupe d'arcades comprenait des motifs empruntés au cloître du monastère de Belem et un deuxième groupe d'arcades, des travées du cloître gothique de l'abbaye de Batalha.

25. Arch. adm. B.n.F., 2011/001/10005, correspondance des architectes, 1879, grands travaux, lettre de Pascal au ministre des travaux publics, 5 janvier 1879. Pascal invoque, outre son talent, une sorte de dette de reconnaissance qu'a le ministre des travaux publics envers Degeorge, car pour le monument à Regnault, le sculpteur est juste rentré dans ses frais.

26. Ils avaient créé la « Société en nom collectif Watrinelle et Germain » en 1869 ; elle fut dissoute en 1881 après 24 chantiers menés en commun. Je remercie vivement M. MichelGasse, qui a travaillé sur la biographie de Gustave Germain, pour ces renseignements.

27. Ce seront ces moulages notamment que Pascal proposera à Camille Enlart, directeur du musée de sculpture comparée du Trocadéro. Pour les extérieurs il s'agit surtout de moulages de sculptures de la cour d'honneur, place Louvois; pour les intérieurs, il s'agit de la salle reconstituée du cabinet des médailles sur la rue Vivienne où a eu lieu l'exposition des Primitifs en 1904 et aussi de la galerie Mazarine. Arch. adm. B.n.F., 2011/001/10011, lettre de Pascal au directeur des bâtiments civils et palais nationaux, 15 novembre 1904. Enlart remercie Pascal de ce don dans une lettre qu'il lui envoie des États-Unis où il a constaté sa popularité : «Je viens d'avoir le plaisir de causer de vous en Amérique avec plusieurs architectes, [écrit-il à Pascal] notamment avec M. Despradelle, chez qui j'ai diné à Boston, avec MM. Masqueray et Carrère, qui m'ont invité à New York. 
Vous avez, ce qui ne m'étonne pas, de bons amis et de grands admirateurs dans ce pays que je viens de parcourir ». Ibid., lettre de Camille Enlart à Pascal, $1^{\mathrm{er}}$ novembre 1904.

28. Arch. adm. B.n.F., 2011/001/10005, B.N., correspondance des architectes, 1879, grands travaux, bâtiment neuf sur la rue Colbert, façade sur la grande cour, soumission pour l'exécution des travaux de sculpture, Watrinelle et Germain, 21 janvier 1879. Le devis des travaux de sculpture indique : «Au rez-de-chaussée, la pierre, demi-dure, de banc royal présente un motif de la baie du milieu avec tête et coquille, quatre motifs des baies latérales avec des trophées des arts, trois motifs de tête avec chute tombant sous l'archivolte, deux chapiteaux de pilastres ioniques, deux chapiteaux de pilastres ioniques avec retour d'angle.

$\mathrm{Au} 1^{\mathrm{er}}$ étage, on a de la pierre tendre, de l'abbaye du Val. On a deux chapiteaux pilastres composites sous le grand fronton, deux chapiteaux pilastres composites avec retour d'angle, un grand motif coquille à la baie du milieu, des motifs coquilles avec figures à droite et à gauche, des motifs de coquilles et cornes d'abondance ou branches, des fenêtres à droite et à gauche, des consoles aux fenêtres de la galerie ».

29. Arch. adm. B.n.F., 2011/001/10005, Bibliothèque nationale, correspondance des architectes, 1879, grands travaux, lettre du directeur des bâtiments civils et palais nationaux à Pascal, 19 mars 1879.

30. Léon Labrouste, «Bibliothèque nationale à Paris. Bâtiments sur la cour d'honneur ", op. cit., p. 3-4.

31. Ils accueilleront la salle de lecture du département des manuscrits.

32. Arch. adm. B.n.F., 2011/001/10005, grands travaux, 1879, lettre de Pascal au ministre, octobre 1879.

33. Ève Netchine et Edmée Srauch,» La salle B », Mélanges autour de l'histoire des livres imprimés et périodiques, op. cit., p. 243-244.

34. Rapport du 27 mars 1858 demandé à Prosper Mérimée, président d'une commission constituée par Achille Fould pour étudier les modifications à introduire dans l'organisation de la Bibliothèque impériale.

35. Ministre des finances entre 1849 et 1852, ministre d'État du 28 juillet 1852 au 23 novembre 1860, puis à nouveau ministre des finances de 1861 à 1867.

36. Simone Balayé, «Les publics de la Bibliothèque nationale », Histoire des bibliothèques françaises. Les bibliothèques de la Révolution et du XIX ${ }^{e}$ siècle : 1789-1914, Paris, éd. du Cercle de la Librairie, 1991, 671 p., p. 330.

37. Ève Netchine et Édmée Strauch, «la salle B», Mélanges autour de l'histoire des livres imprimés et périodiques, op. cit., p. 249-250.

38. Arch. adm. B.n.F., 2011/001/10006, B.N., correspondance des architectes, grands travaux, 1881.

39. Pour la salle de la rue Colbert et ses dépendances, Pascal commande 100 chaises ( 35 fr.) et 40 fauteuils (45 fr.) à G. Quignon. Arch. adm. B.n.F., 2011/001/10006, lettre de Pascal au ministre, 9 mai 1880.

40. «En vertu du nouveau règlement arrêté le 5 mai par Son Excellence le Ministre de l'Intérieur, la salle est ouverte tous les jours même le dimanche de $10 \mathrm{~h}$. à $4 \mathrm{~h}$. pour toute personne agée de seize ans accomplis. L'entrée provisoire, comme le local qui va d'abord être affecté à cette salle, sera rue Colbert, $n^{\circ} 3$ ». Moniteur universel, 16 mai 1878, B.n.F., dp. Mss, N.a.fr. 13026, f. 279. 
41. Le Livre. Revue mensuelle, Paris, $2^{\mathrm{e}}$ partie, Bibliographie moderne, 1881, p. 118-119, cité par Ève Netchine et Édmée Strauch, «la salle B », Mélanges autour de l'histoire des livres imprimés et périodiques, op. cit., $\mathrm{n}^{\circ} 24$ p. 249.

42. Pascal pensait à attribuer par la suite au département des manuscrits, jusque-là encore logé dans l'hôtel Tubeuf, l'emplacement de cette salle de lecture ( $1^{\text {er }}$ étage de la moitié ouest de l'aile sur la rue Colbert), plus le $1^{\text {er }}$ étage de l'aile à construire à la suite sur la $2^{\mathrm{e}}$ partie de la rue Colbert, et enfin le $1^{\mathrm{er}}$ étage de l'aile du fond de la cour d'honneur, réalisant une sorte de " $T$ » entièrement consacré aux manuscrits. Les magasins de manuscrits seraient logés dans les bâtiments donnant sur la rue Colbert et la salle de travail des manuscrits dans l'aile est sur la cour d'honneur.

43. Arch. adm. B.n.F., 2011/001/10006, lettre de Delisle à Pascal, 19 juin 1880.

44. Le Livre, op. cit., p. 118, cité par Ève Netchine et Édmée Strauch, Mélanges autour de l'histoire des livres imprimés et périodiques, op. cit., $\mathrm{n}^{\circ} 41$ p. 251.

45. «J'aurais été enchanté de pouvoir faire un essai d'éclairage électrique dans la salle que nous devons ouvrir ». Lettre de l'architecte à l'administrateur, 21 novembre 1880, B.n.F., dp. Mss, Archives modernes, 229, 12, cité par Ève Netchine et Édmée Strauch, Mélanges autour de l'histoire des livres imprimés et périodiques, op. cit., nº 23 p. 247.

46. Arch. adm. B.n.F., 2011/001/10006, grands travaux, 1880, lettre de Pascal à Delisle, 10 janvier 1880.

47. Arch. nat. $\mathrm{F}^{21} 2995$, Bibliothèque nationale, exercice 1896, chapitre 42, grosses réparations des bâtiments civils et travaux pour l'exercice 1896 .

48. Arch. adm. B.n.F., 2011/001/10007, état sommaire des propositions faites pour les travaux de réfections et de grosses réparations à exécuter pendant l'exercice 96. Pascal songe déjà à la future salle Ovale.

49. Arch. adm. B.n.F., 2011/001/10075, lettre de Pascal au sous-secrétaire d'État aux beaux-arts, 21 octobre 1897.

50. «Des escaliers branlants, des portes mal jointes qui semblent empruntées à une agence de démolition, une salle qui a l'air d'un chauffoir de maison centrale, voilà ce que le premier établissement de France offre aux pauvres gens, aux ouvriers, au grand public en un mot.» B.n.F., dp. Mss, N.a.fr. 13027, f. 15 : Édouard Drumont, » La Bibliothèque nationale ", La Liberté, 30 mars 1885.

51. Léon Labrouste, » Bibliothèque nationale, rue de Richelieu à Paris ", R.G.A.T.P., $\mathrm{n}^{\circ}$ XXXV, 1878, p. 147, note 3.

52. Arch. nat. $\mathrm{F}^{21} 5855$, ministère des travaux publics, bâtiments civils, agence des travaux de la Bibliothèque nationale, cabinet de l'architecte, 10 octobre 1879.

53. Henry Marcel, Henri Bouchot, Ernest Babelon, Paul Marchal, Camille Couderc, La Bibliothèque nationale, op. cit., p. 8.

54. Henri Bouchot, «Les derniers travaux de décoration exécutés à la Bibliothèque nationale par M. Pascal, architecte », op. cit., p. 95.

55. Une lettre du 3 mars 1879 du directeur des bâtiments civils et palais nationaux à Pascal, l'informe du remplacement de Duc, décédé, par André comme inspecteur général des bâtiments civils, par arrêté du 4 février 1879. Arch. adm. B.n.F., 2011/001/10005, grands travaux, 1879 .

56. Arch. nat., $\mathrm{F}^{21}$ 5856, 25 novembre 1879. 
57. Jules André, ancien élève des Beaux-Arts, de Huyot (1780-1840) et d'Hippolyte Lebas (1782-1867), grand prix de Rome en 1848, avait travaillé à la reconstruction de la Bibliothèque impériale sous les ordres de Labrouste et lui avait succédé à la tête de l'atelier créé par ce dernier en 1830.

C'est au fauteuil de Jules André que Pascal sera nommé à l'Institut le 3 mai 1890. Archives de l'Institut, registre des procès-verbaux, 2E18.

58. Henri Bouchot, «Les derniers travaux de décoration exécutés à la Bibliothèque nationale par M. Pascal, architecte », op. cit., p. 94-95.

59. La restauration de la charpente du vieux bâtiment du fond de la cour d'honneur est due à l'entrepreneur G. Dubief. Arch. nat., $\mathrm{F}^{21}$ 5857, lettre de Pascal du 2 août 1882.

60. Le sommaire des travaux de réparation du vieux bâtiment au fond de la cour de la place Louvois indique (Arch. adm. B.n.F., 2011/001/10005, grands travaux, 1879) :

Transformation des planchers en bois en planchers en fer avec solives assemblées dans les poutres pour les deux étages, avec solives portées sur les poutres pour l'étage de cave.

Dans l'étage du comble, parquet à l'anglaise, casiers et planchers à claire-voie comme dans le nouveau bâtiment Colbert.

$\mathrm{Au} 1^{\mathrm{er}}$ étage, réparation et replacement du parquet à panneaux qui y figure. Restauration de toutes les armoires, de la galerie, des balustrades en fer ; complément d'installation du côté des fenêtres avec casiers, balcons, etc. fourniture et réemploi de vieilles boiseries et sculptures, notamment de consoles et d'ébrasement et de montants sculptés.

61. Arch. adm. B.n.F., 2011/001/10006, B.N., correspondance des architectes, grands travaux, 1881.

62. L'entrepreneur Tabary chargé de la restauration, indique que Lefuel a utilisé ce procédé de ciment métallique pour restaurer le Louvre et les Tuileries, notamment la colonnade. Arch. nat., $\mathrm{F}^{21}$ 5857, B.N., grosses réparations, 1881, lettre de Pascal au ministre des arts, Antonin Proust, le 4 déc. 1881. Cela est confirmé par une lettre de son associé Humbert Droz, datée du 4 octobre 1878, qui déclare utiliser le procédé de fabrication du ciment métallique mis au point par son ancien associé J. Fontenelle, et qui indique l'avoir utilisé pour réparer le pavillon Mollien au Louvre en 1874, et "plus récemment » à la Bibliothèque nationale. Arch. nat., $\mathrm{AJ}^{64}, 214$, Agence du palais des Tuileries et du Louvre, 1874, correspondance officielle, lettre de Humbert Droz au ministre des travaux publics, 4 octobre 1878.

63. Arch. nat., $\mathrm{F}^{21} 5855$, B.N., agence des travaux de la B.N., cabinet de l'architecte, le 10 octobre 1879.

64. Arch. adm. B.n.F., 2011/001/10006, lettre de Pascal au ministre des travaux publics, 30 octobre 1881 et

Arch. nat., $\mathrm{F}^{21}$ 5857, B.N., grosses réparations, 1881, lettre de Pascal au ministre des arts Antonin Proust, le 4 décembre 1881.

65. Arch. nat. $\mathrm{F}^{21} 6536$, avis et rapports du conseil des bâtiments civils, séance du 10 juin 1896, rapporteur : Pascal.

66. Le 12 décembre 1881, Tabary s'engage à effectuer les travaux de ciment métallique en vue de la restauration du bâtiment du fond de la cour d'honneur pour une somme de 2000 fr. qui comprenait non seulement l'application du ciment frais mais aussi la réfection de portions de sculpture. Arch. adm. B.n.F., 2011/001/10006, lettre du ministre des arts à Pascal. 
67. Arch. adm. B.n.F., 2011/001/10006, lettre de Pascal au ministre des travaux publics, 30 octobre 1881.

68. Arch. adm. B.n.F., 2011/001/10007, lettre de Pascal au ministre, 10 juin 1894.

69. La stucatine pour la cour de la reliure a été fournie par Collantier successeur de Doix, 10 rue Poisson.

70. Arch. adm. B.n.F., 2011/001/10009, lettre de Pascal au ministre de l'instruction publique et des beaux-arts, 21 juin 1901.

71. L'entrepreneur est C. Fouinat, 170 quai Jemmapes.

72. Arch. nat., $\mathrm{F}^{21} 5855$, B.N., année 1880 , agence des travaux de la B.N., cabinet de l'architecte J.-L. Pascal, le 10 oct. 1879.

73. Arch. adm. B.n.F., 2011/001/10006, lettre de Pascal au ministre de l'instruction publique et des beaux-arts, 4 novembre 1882 .

74. «L'œuvre de Scheffer ne resta pas longtemps au cabinet. En 1823, le comte de Forbin la réclama pour une autre administration et et la fit remplacer par une copie du tableau du baron Gérard exécutée par Melle Duvidal [Louise Rose Julie Duvidal de Montferrier (1797-1869), peintre d'histoire, élève de Gérard]. Au moment de la Révolution de 1830, ce deuxième Louis XVIII disparut à son tour des cadres dorés du Cabinet. Il fut roulé et rangé dans les combles, d'où il partit au XXe siècle pour le musée de Versailles. On mit à sa place une copie du Louis XV de Van Loo, visible encore aujourd'hui.» Thierry Sarmant, Le Cabinet des médailles de la Bibliothèque nationale, 1661-1848, Paris, École des Chartes, 1994, 403 p., p. 325.

75. Arch. adm. B.n.F., 2011/001/10006, lettre de Pascal au ministre de l'instruction publique et des beaux-arts, 4 novembre 1882 .

76. Ibid., lettre de Pascal au directeur des bâtiments civils et palais nationaux, 19 juillet 1888.

77. Ibid., lettre de Pascal au ministre de l'instruction publique et des beaux-arts, 4 novembre 1882.

78. Henri Bouchot, «Les derniers travaux de décoration exécutés à la Bibliothèque nationale par M. Pascal, architecte », op. cit., p. 96.

79. Dans ce qui constituait autrefois la salle à manger de la marquise de Lambert. Pascal l'écrit quand, devant détruire l'ancien bâtiment des globes situé derrière le bâtiment de la cour d'honneur, il en réalise des moulages:»La curiosité seule aurait suffi à justifier cette opération si je n'avais eu en outre à y ajouter, en vue de la reconstitution de l'ancienne salle du cabinet des médailles dans la nouvelle installation, des parties de corniches, cartouches et détails encore en place dans mon agence [située dans l'hôtel Colbert], dernier reste subsistant de l'ancien Cabinet de France ». Arch. adm. B.n.F., 2011/001/10009, lettre de Pascal au ministre, 26 novembre 1901.

80. Arch. adm. B.n.F., 2011/001/10006, lettre à Pascal du ministre de l'instruction publique et des beaux-arts, 18 juillet 1882 .

81. Antiquaire passionné, Gaston d'Orléans avait réuni dans son château de Blois et son palais du Luxembourg, une incomparable collection de gemmes gravées et d'antiques, de singularités exotiques, qu'il légua en 1660 à son neveu Louis XIV.

82. La bibliothèque de Daniel Huet, évêque d'Avranches, ancien précepteur du grand dauphin, contenant plus de 8000 livres imprimés fut cédée au roi Louis XV en échange d'une pension de 1750 livres par M. de Charsigné, un des héritiers du prélat. 
83. C'est Leclercq qui fournit les 4 gaines en brèche violette de Seravezza pour 4000 fr. en tout. Arch. adm. B.n.F., 2011/001/10011.

84. Sculpture envoyée de Rome par Sicard en 1893 ou 1895, alors que l'artiste était pensionnaire à la Villa Médicis; elle fut achetée par l'avocat Scheikevitch qui deviendra le beau-père de Sicard puis donnée par lui à la Bibliothèque nationale qui déposa l'oeuvre en 1948 à la mairie de Tours; le musée des beaux-arts de Tours possède le modèle en plâtre de cette statue dont il existe des reproductions réduites en biscuit de Sèvres et en marbre. Hauteur : $130 \mathrm{~cm}$., largeur : $80 \mathrm{~cm}$., profondeur : $72 \mathrm{~cm}$. Dossier base Mérimée, référence : IM37000818, notice rédigée par Vincent Droguet et Marie-Thérèse Réau.

85. Pascal demandait qu'y soient placés deux hautes vitrines de bois doré, deux meubles isolés avec vitrines de bois doré, quatre sièges de bois sculpté, quatre gaines de marbre de brèche violette de Serravezza (fournies, elles, en 1905) pour y supporter les bustes de Jean de Berry, Gaston d'Orléans (côté est) et Henri Estienne et Daniel Huet (côté ouest), ainsi que deux consoles. Arch. adm. B.n.F., 2011/001/10009, état sommaire des propositions faites pour les travaux de réfections et de grosses réparations à exécuter pendant l'exercice 1901, renouvelé chaque année jusqu'en 1910: Arch. adm. B.n.F., 2011/001/10025, état sommaire des propositions faites pour les travaux de réfections et de grosses réparations à exécuter pendant l'exercice 1911, fait par Pascal, le 26 octobre 1910.

86. Arch. nat., $\mathrm{F}^{21} 5863$, B.N., décoration du vestibule d'honneur, lettres du ministre de l'instruction publique au ministre des travaux publics, 13 octobre 1882 et 25 mai 1892. La Science sera livrée le 31 mars 1892 ; elle mesure $4,12 \mathrm{~m}$. dans sa hauteur et 2,50 m. dans sa largeur; son prix est de 48 414,24 francs. La Mécanique sera livrée le 4 janvier 1892 et La Poésie, le 22 octobre 1892. Arch. adm. B.n.F., 2011/001/10343, lettre de Gerspach à l'administrateur général de la Bibliothèque nationale, 29 mars 1892 et lettres du ministre des travaux publics à Pascal, 3 juin 1892 et 21 octobre 1892.

87. Arch. adm. B.n.F., 2011/001/10006, lettre de Marcel à Pascal, 24 octobre 1882.

88. Henry Marcel, Henri Bouchot, Ernest Babelon, Paul Marchal, Camille Couderc, La Bibliothèque nationale, op. cit., p. 15.

89. Arch. nat., $\mathrm{F}^{21} 2995$, lettre de Pascal au ministre de l'instruction publique et des beauxarts, 18 décembre 1885.

90. G. et R. Hamot et $C^{\text {ie }}$ s'engagent à fournir les tapis nécessaires pour l'antichambre et le cabinet de l'administrateur de la B.N. Arch. adm. B.n.F., 2011/001/10006, avis de l'approbation d'une soumission du directeur des bâtiments civils et palais nationaux à Pascal, 4 janvier 1886.

91. Arch. nat., $\mathrm{F}^{21}$ 2995, lettre de Léopold Delisle à Jean-Louis Pascal, 3 septembre 1887.

92. Henri Bouchot, «Les derniers travaux de décoration exécutés à la Bibliothèque nationale par M. Pascal, architecte », op. cit., p. 96.

93. Le crédit alloué était de 373000 francs. Arch. nat., $\mathrm{F}^{21}$ 5855, B.N., édifices publics, 1880 , chapitre 54, rapport du premier bureau de la direction des bâtiments civils au ministre des travaux publics, le 19 mars 1880.

94. «Les anciens "cabinets" du Cardinal Mazarin, dans l'aile qui sépare la cour de la rue Neuve-des-Petits-Champs et la cour de la rue Vivienne [hôtel Tubeuf], sur lesquelles ils prennent jour au midi et au nord, sont devenus trop étroits pour l'affluence des travailleurs. La salle publique des manuscrits doit être installée prochainement dans l'aile 
du fond de la cour, parallèle à la rue de Richelieu et qu'occupait, il y a vingt ans, la salle publique de lecture des imprimés au premier étage.» Arch. nat., $\mathrm{F}^{21}$ 5863, (titre: «Couverture de la grande cour. B.N. »). An 1882-1886, article du Figaro, vendredi 6 juillet 1883, Augustin Vitu.

95. Henri Bouchot, «Les derniers travaux de décoration exécutés à la Bibliothèque nationale par M. Pascal, architecte », op. cit., p. 96.

96. Arch. nat., $F^{21}$ 5857, marchés 1881-1896, année 1882, lettre de Pascal à Jules Ferry, ministre de l'instruction publique et des beaux-arts, 18 juin 1882 et Arch. adm. B.n.F., 2011/001/10006, lettre de Pascal à Jules Ferry.

97. Le directeur des bâtiments civils et palais nationaux approuve le 22 juillet 1882 la soumission aux termes de laquelle G. Germain s'engage à exécuter pour $50000 \mathrm{fr}$. la restauration du bâtiment du fond de la cour d'honneur : « en vue de la restauration des menuiseries et décorations artistiques». Arch. adm. B.n.F., 2011/001/10006, lettre à Pascal.

98. Pour la réfection de la corniche de la grande salle du $1^{\text {er }}$ étage du bâtiment au fond de la cour d'honneur, Germain, sculpteur ornemaniste, s'engage à exécuter les travaux de sculpture et de moulage que comporte la réfection dont il s'agit pour $3000 \mathrm{fr}$., Arch. adm. B.n.F., 2011/001/10006, lettre à Pascal du ministre des arts, 2 décembre 1881.

99. Jean-Pierre Babelon, Chantilly, Paris, éd. Scala, 1999, 247 p.

100. Arch. adm. B.n.F., 2011/001/10006, lettre au ministre de Pascal, juin 1882.

101. A. M. Bordeaux, $6872 \mathrm{M} 33$, lettre de Pascal au maire, 21 août 1885.

102. Arch. adm. B.n.F., 2011/001/10006, lettre de Ronsin à Pascal, 18 octobre 1882.

103. Arch. adm. B.n.F., 2011/001/10002, mémoire des ouvrages de menuiserie exécutés à la Bibliothèque nationale d'après les ordres de $\mathrm{M}$. Pascal.

104. Arch. adm. B.n.F., 2011/001/10006, lettre du vérificateur G. Herny, 20 février 1882.

105. Il ne reste alors à la Bibliothèque que deux parquets en feuilles. Pascal acceptera de poser un linoléum dans la salle des manuscrits en avril 1912, car le parquet est alors en mauvais état et que » cela cachera plutôt sa misère ", selon le mot de Pascal. Arch. adm. B.n.F., 2005/028/357, correspondance des architectes, 1896-1899.

106. Léon Labrouste, La Bibliothèque nationale, son début et ses accroissements, ses bâtiments et ses constructions, ses agrandissements, ses travaux, op. cit., p. 28.

107. Ibid., p. 29-30.

108. B.n.F., dp. Est., Ha 132, «La restauration de la Bibliothèque nationale : la nouvelle salle des manuscrits ", L'Illustration, 6 novembre 1886, n 2280-309.

109. Arch. adm. B.n.F., 2011/001/10006, lettre de Mortreuil à Pascal, 27 novembre 1882.

110. L'ébéniste Auguste Blanqui, 8 rue Cherchell, Marseille, était médaillé d'argent de l'exposition universelle de 1878 à Paris, et avait reçu un diplôme d'honneur lors d'une exposition en 1879 à Marseille. Il réalise à partir de 1893 tous les aménagements intérieurs, l'ensemble des boiseries, les huisseries, et tous les meubles du château d'Avignon à Sainte-Marie-de-la-Mer (Bouches-du-Rhône), pour l'industriel et négociant Louis Noilly-Prat (1845-1932).

111. «La bonne exécution de meubles que j'ai vus en différents endroits à Paris par M. Blanqui me fait désirer de le voir mettre en concurrence avec les grands ébénistes parisiens, auxquels il conviendrait de proposer une adjudication restreinte, sur la base 
des plans et des modèles que j'ai fait préparer ", Arch. adm. B.n.F., 2011/001/10006, lettre de Pascal au ministre de l'instruction publique et des beaux-arts, 29 novembre 1882.

112. Henry Auguste Fourdinois succède en 1867 à son père Alexandre Georges Fourdinois (1799-1871) à la tête de la grande maison d'ébénisterie du Second Empire.

113. Redond, 21 rue du faubourg Saint Antoine, Paris.

114. Arch. adm. B.n.F., 2011/001/10006, lettre de Pascal au ministre de l'instruction publique et des beaux-arts, 29 novembre 1882 .

115. Arch. nat., $\mathrm{F}^{21} 5857$, travaux de restauration, chapitre 51 : exercice 1885, soumission. Fourdinois demeure au n ${ }^{\circ} 46$ de la rue Amelot, à Paris (11 $\left.{ }^{\mathrm{e}}\right)$.

116. «En avril 1886, [ai livré] cent fauteuils Louis XV chêne, siège et dos canné, du département des manuscrits ». Arch. adm. B.n.F., 2011/001/10008, lettre de Quignon à Pascal, 17 mars 1898.

117. Henri Bouchot, «Les derniers travaux de décoration exécutés à la Bibliothèque nationale par M. Pascal, architecte », op. cit., p. 96.

118. Ibid.

119. B.n.F., dp. Est., Ha 132, «La restauration de la Bibliothèque nationale : la nouvelle salle des manuscrits ", L'Illustration, 6 novembre 1886, n 2280-309.

120. La statue de l'empereur Charles VI en » Hercules Musarum» est signée Peter (1660-1714) et Paul Strudel (1648-1708).

121. La coupole située à $30 \mathrm{~m}$. de hauteur est ornée d'une fresque représentant l'apothéose de l'empereur Charles VI. Le programme de la décoration des fresques fut conçu par le savant de la cour Conrad Adolph von Albrecht (1682-1751).

122. Henri Bouchot, "Les derniers travaux de décoration exécutés à la Bibliothèque nationale par M. Pascal, architecte », op. cit., p. 96.

123. Ibid., p. 97.

124. Ibid., p. 95.

\section{RÉSUMÉS}

Lorsque Jean-Louis Pascal hérite du poste d'architecte en chef de la Bibliothèque nationale, en juillet 1875, le pavillon situé à l'angle des rues Richelieu et Colbert et les deux premières travées de l'aile nord de la cour d'honneur ont déjà été démolis par Henri Labrouste. Le problème se pose de savoir si l'on continue à reconstruire ce bâtiment hérité du XVIII ${ }^{\mathrm{e}}$ siècle comme Labrouste l'avait prévu ou si l'on le restitue dans son état initial. Des projets mixtes envisageant une reconstruction du bâtiment en y insérant des motifs architecturaux dus à Labrouste sont balayés par le conseil des bâtiments civils qui exige une reconstruction à l'identique. Pascal installe au $1^{\text {er }}$ étage de l'aile nord restituée sur la cour d'honneur la salle publique de lecture qui fonctionnera de 1881 à 1936. À l'est, le bâtiment dû à Claude Armand Mollet et Robert de Cotte est totalement restauré par Jean-Louis Pascal, qui y emploie les matériaux nouveaux que sont le fer et le ciment métallique. Il y installe au rez-de-chaussée le vestibule d'honneur et à l'étage, la salle de lecture des manuscrits ouverte en 1886. Pour décorer ces deux pièces, il reprend les tableaux qui 
ornaient l'ancien cabinet des médailles de l'arcade Colbert détruit en 1865 et fait reconstituer par son sculpteur favori Gustave Germain moulures et boiseries anciennes, en moulant les motifs sculptés du XVIII ${ }^{\mathrm{e}}$ siècle dont il restait quelques exemples à la Bibliothèque nationale notamment dans son agence située au $\mathrm{n}^{\circ} 12$ de la rue Colbert, dernier reste de l'hôtel de la marquise de Lambert transformé en cabinet des médailles en 1741.

When Jean-Louis Pascal became the chief architect of the National Library in July 1875, the pavilion located at the intersection of the Rue de Richelieu et Rue Colbert and the first two spans of the Northern wing of the Honour Courtyard have already been demolished by Henri Labrouste. The issue is whether the architect keeps rebuilding this XVIII ${ }^{\text {th }}$ century building, as Labrouste had planned to do, or restores it in his original state. Mixed projects combining reconstruction with architectural patterns designed by Labrouste have been swept away by the "Bâtiments civils" Council, who requires a similar reconstruction. Pascal sets at the $1^{\text {st }}$ floor of the Northern wing rebuild on the Honour Courtyard the public Reading room to be used from 1881 to 1936 . On the Eastern Side, the building due to Claude Armand Mollet and Robert de Cotte is completely restored by Jean-Louis Pascal, who uses new materials such as the iron and metallic cement. On the ground floor takes place the main hall and on the first floor the Manuscripts Reading room (opened in 1886). To adorn these two rooms, he hangs on the walls some paintings which used to be at the former Cabinet des médailles of the Arcade Colbert demolished in 1865. He also asks his favorite sculptor Gustave Germain to reconstitute ornaments and old woodwork, by moulding some XVIII ${ }^{\text {th }}$ century patterns whose a few of them still stayed at the National Library, especially in his agency located Rue Colbert $\left(n^{\circ} 12\right)$, last testimony of the marquise de Lambert's mansion transformed into Cabinet des médailles en 1741.

Als Jean-Louis Pascal im Juli 1875 die Stelle des Architecte en chef an der Bibliothèque Nationale übernimmt, sind bereits der an der Ecke der Rue Richelieu und der Rue Colbert gelegene Pavillon sowie die ersten beiden Joche des Nordflügels des Ehrenhofes von Labrouste entfernt worden. Daher stellt sich zunächst für Pascal die Frage, ob die Rekonstruktion der Gebäudeteile aus dem 18. Jahrhundert wie von Labrouste vorgesehen fortgeführt werden soll oder ob das Ensemble in seinen ursprünglichen Zustand zurückgeführt werden soll. Der Conseil des Bâtiments civils äußerst sich ablehnend gegenüber dem Vorschlag einer Rekonstruktion des Gebäudes unter Einbeziehung architektonischer Motive Labroustes und fordert stattdessen eine Rekonstruktion des Originalzustandes. Pascal errichtet daraufhin im ersten Geschoss des wiederhergestellten Nordflügels zum Ehrenhof hin den öffentlichen Lesesaal, der von 1881 bis 1936 genutzt wird. Auf der östlichen Seite des Komplexes wandelt Pascal das von Claude Armand Mollet und Robert de Cotte errichtete Gebäude völlig um und verwendet dort neue Materialien wie Eisen und Stahlbeton. Dort richtet Pascal im Erdgeschoss das Ehrenvestibül sowie in der ersten Etage den Handschriften-Lesesaal ein, der 1886 eröffnet wird. Zur Ausschmückung dieser Räumlichkeiten greift Pascal auf Gemälde zurück, die zuvor das Cabinet des Médailles von Colbert geschmückt haben und deren hölzerne Rahmen und Verzierungen er durch seinen bevorzugten Bildhauer Gustave Germain wiederherstellen lässt, wobei er Motive aus dem 18. Jahrhundert wiederaufgreift, von denen einige Vorbilder in der Bibliothèque Nationale erhalten waren, insbesondere in seinen Büroräumen, die er in der Rue Colbert Nr. 12 eingerichtet hatte, im letzten Überbleibsel des 1741 in die Münzsammlung verwandelten vormaligen Hôtel particulier der Marquise de Lambert. 
INDEX

Index chronologique : époque contemporaine, XIXe siècle

Mots-clés : bibliothèque, restauration, restitution

Keywords : library, restoration, restitution

Schlüsselwörter : Bibliothek, Restaurierung, Wiederherstellung

\section{AUTEUR}

\section{ANNE RICHARD-BAZIRE}

Anne Richard-Bazire est docteur de l'École pratique des hautes études (section des sciences historiques et philosophiques), diplômée en histoire de l'art contemporain et en archéologie égyptienne. Elle est actuellement chargée du cours de synthèse de l'Histoire de l'architecture occidentale, $\mathrm{XIX}^{\mathrm{e}}-\mathrm{XX}^{\mathrm{e}}$ siècles, à l'École du Louvre. Elle a rédigé différents articles : «L'habitat néo-gothique à Paris au XIX ${ }^{\mathrm{e}}$ siècle », La demeure médiévale à Paris, Étienne Hamon et Valentine Weiss (dir.), Paris, 2012, Archives Nationales, p. 250-257 ; « La salle Ovale ou l'histoire des dysfonctionnements dans les chantiers parisiens du XIX siècle à travers l'exemple de la Bibliothèque nationale ", La Collégialité et les dysfonctionnements dans la décision administrative, JeanMichel Leniaud et François Monnier (dir.), Paris, 2011, École Pratique des Hautes Études, p. 103-112 ; «Jean-Louis Pascal ou le respect du patrimoine », article mis en ligne sur le site "Archicab", le 4 décembre 2011, http://www.jeanfrancoiscabestan.com/bnfrichelieu.html ; « Le concours pour la reconstruction de l'Hôtel de Ville de Paris (1872-1873), un échec pour l'architecte Jean-Louis Pascal ", Livraisons d'histoire de l'architecture, ${ }^{\circ}$ 19, 2010, p.111-134 ; « L'escalier d'honneur de la Bibliothèque nationale de la rue de Richelieu », Sites et Monuments, $\mathrm{n}^{\circ}$ 207, oct.-nov.-déc. 2009, p. 22-24 ; « La salle Ovale », Revue de la Bibliothèque nationale de France, $\mathrm{n}^{\circ}$ 30, 2008, 96 p., p. 32-39 ; « La faculté de médecine et de pharmacie de Bordeaux par Jean-Louis Pascal (1876-1888 et 1902-1922) », Livraisons d'histoire de l'architecture, $\mathrm{n}^{\circ} 13,2007, \mathrm{p} .105-120$; « Le ministère de l'agriculture de la rue de Varenne par Emmanuel Brune, l'alliance de la science et de l'art ", Livraisons d'histoire de l'architecture, $\mathrm{n}^{\circ}$ 8, 2004, p. 81-94; « Le parvis de Notre-Dame » et « Le square Jean XXIII ", Autour de Notre-Dame, sous la dir. de Alain Erlande-Brandeburg, Jean-Michel Leniaud, François, Christian Michel, Paris, D.A.A.V.P., 2003, 269 p., p. 240-242 et 243-245 ; « Les réalisations de Pascal ", et "L'École des beaux-arts » avec Fabienne Doulat, Les bibliothèques parisiennes, architecture et décor, sous la dir. de Myriam Bacha et Christian Hottin, Paris, D.A.A.V.P., 2002, 272 p., p. 115-117 et 132-134; « Un siècle de réflexion sur la construction des bibliothèques ", Des palais pour les livres, Labrouste, Sainte-Geneviève et les bibliothèques, sous la dir. de Jean-Michel Leniaud, Paris, éd. Maisonneuve \& Larose, 2002, 189 p., p. 58-69 ; « Jean-Louis Pascal et la création de la salle des périodiques de la Bibliothèque nationale (1883-1936) ", Livraisons d'histoire de l'architecture, $\mathrm{n}^{\circ}$ 1, 2001, p. 105-125. Elle a dirigé avec Martin Drouin : La Sélection patrimoniale, Québec, Éditions MultiMondes, coll. Cahiers de l'Institut du patrimoine de l'UQAM, n 13, 2011, 388 p. Elle a participé en octobre 2001 au colloque international organisé par la Bibliothèque Sainte-Geneviève à Paris : « Henri Labrouste et la Bibliothèque Sainte-Geneviève, Histoire et actualité », en octobre 2009 au colloque : «Victor Laloux, un architecte dans sa ville » organisé à Tours par le Conseil général d'Indre-et-Loire, en juillet 2010 aux journées d'études organisées par le Centre-Ledoux-Université Paris I, la Commission du Vieux Paris et l'Association pour la Sauvegarde et la Mise en valeur du Paris Historique, sur le thème : «BNF Richelieu : un projet en 
questions »; en octobre 2010, elle a été la coordinatrice scientifique avec Martin Drouin de la Sixième Rencontre internationale des jeunes chercheurs en patrimoine sur le thème de la " Sélection patrimoniale ", organisée conjointement par l'Université du Québec à Montréal et l'École des Chartes à Paris, sous la direction de Luc Noppen, Lucie K. Morisset (UQAM) et JeanMichel Leniaud (École des chartes). Elle a participé en janvier 2011 aux Journées annuelles de l'équipe Histoire du droit public et de l'administration, EPHE, sous la direction de François Monnier, directeur de l'UR, Jean-Michel Leniaud et Jean-Miguel Pire sur le thème : « Les dysfonctionnements dans la décision administrative ", en novembre 2011 à la journée d'études de l'équipe Histara de l'EPHE, organisée à l'INHA par Jean-François Belhoste, directeur d'études à l'EPHE, et Isabelle Parizet, maître de conférences à l'EPHE, sur le thème : « Architectures bancaires : entre le Beau et l'Utile », en décembre 2012 au colloque international organisé par l'INHA, Le musée d'Orsay et la Maison des centraliens : « William Le Baron Jenney (1832-1907). De l'École centrale de Paris aux gratte-ciel de Chicago ». Elle a réalisé de mars 2012 à janvier 2013 pour le compte de l'OPPIC les recherches historiques préalables à la restauration des galeries Mansart, Mazarine et de la « chambre de Mazarin » à la Bibliothèque nationale Richelieu sous la direction de l'architecte en chef des monuments historiques A. de Saint-Jouan. Adresse électronique : anne.richardbazire@hotmail.com. 